\title{
Harvey Cushing's craniopharyngioma treatment: Part 1. Identification and clinicopathological characterization of this challenging pituitary tumor
}

\author{
*José María Pascual, MD, PhD, Ruth Prieto, MD, PhD, ${ }^{2}$ and Laura Barrios, BS ${ }^{3}$ \\ 1Department of Neurosurgery, La Princesa University Hospital; ²Department of Neurosurgery, Puerta de Hierro University \\ Hospital; and ${ }^{3}$ Statistics Department, Computing Center, CSIC, Madrid, Spain
}

\begin{abstract}
OBJECTIVE Harvey Cushing (1869-1939) coined the term "craniopharyngioma" (CP) in 1929 to describe a kaleidoscopic group of epithelial tumors involving the hypothalamic-pituitary axis. Throughout his career, he endured a long struggle to accurately diagnose and safely remove these complex lesions, and his resulting surgical series has never before been analyzed in depth. The authors here conduct such an analysis.
\end{abstract}

METHODS In this study, the authors retrospectively examined the CP patient records available in the Cushing Brain Tumor Registry, as well as those CP cases reported by Cushing in medical monographs and scientific reports.

RESULTS Cushing's CP series comprises a total of 124 tumors $\left(\mathrm{CP}_{124}\right)$ compatible with a CP diagnosis. Among this series are 92 cases that could be pathologically verified $\left(\mathrm{CP}_{92}\right)$. This subcohort showed a bimodal age distribution $(41 \%$ aged $\leq 19$ years old) and a balanced sex distribution. Clinical evolution up to diagnosis was longer than 3 years in half of the patients. Typical symptoms found at diagnosis were severe headache (94\%), visual deficits (97\%), panhypopituitarism (76\%), psychiatric disturbances (47\%), and abnormal somnolence $(47 \%)$. The highest rate of endocrine deficits occurred in patients younger than 19 years of age $(p<0.001)$, whereas hypothalamic disturbances were observed mainly in adults between 30 and 49 years $(p=0.02$ ). Hydrocephalus was present in $63 \%$ of the patients, predominantly involving the younger subgroup $(p<0.001)$. Preoperative diagnosis was based on clinical signs, funduscopic exams, and skull radiographs, the latter study showing suprasellar calcifications in $64 \%$ of cases. The majority of tumors (61\%) had developed within the third ventricle (3V) or had invaded it. The adamantinomatous histological variant was the predominant one (73\%). Squamous-papillary CPs occurred only in adults older than 40 years of age $(p<0.001)$. Strong CP adherences to the hypothalamus were demonstrated in $63 \%$ of cases. The infundibulo-tuberal and sellar/suprasellar-3V $\mathrm{CP}$ topographies were associated with the highest rates of hypothalamic dysfunction before surgery $(p<0.001)$, surgical hypothalamic injury $(p<0.001)$, and severe postoperative morbidity and/or mortality $(p=0.009)$. Both topographies showed the strongest adherences to the hypothalamus and $3 \mathrm{~V}(p<0.001)$.

CONCLUSIONS Cushing's CP series comprises severely ill patients with tumors in the late stages of progression, with a high rate of tumors developing primarily within the hypothalamus (infundibulo-tuberal CPs) or invading this structure from the sellar/suprasellar regions. Craniopharyngioma topography was the fundamental variable influencing the clinical manifestations, tumor features, and patient outcomes in this series.

https://thejns.org/doi/abs/10.3171/2018.5.JNS18153

KEYWORDS craniopharyngioma; Harvey Cushing; history of pituitary surgery; hypothalamus; infundibulum; third ventricle; pituitary tumors; pituitary gland

Since scarcely any two are precisely alike and since those of similar size and character show such differences in behavior, the course of any given tumor is impossible to foretell.
Harvey Williams Cushing (1869-1939) is considered one of the founding fathers of neurosurgery (Fig. 1A). ${ }^{8} \mathrm{He}$ laid the foundations for the surgical treatment of most intracranial tumors with his groundbreaking monographs on

ABBREVIATIONS BTR = Brain Tumor Registry; $\mathrm{CP}=$ craniopharyngioma; $\mathrm{CP}_{92}=$ Cushing's subcohort of 92 pathologically verified $\mathrm{CPs}$; $\mathrm{CP}_{124}=\mathrm{Cushing}$ 's entire series of $124 \mathrm{CPs} ; \mathrm{DI}=$ diabetes insipidus; IT = infundibulo-tuberal; $3 \mathrm{~V}=$ third ventricle; $3 \mathrm{VF}=3 \mathrm{~V}$ floor.

SUBMITTED January 18, 2018. ACCEPTED May 29, 2018.

INCLUDE WHEN CITING Published online October 5, 2018; DOI: 10.3171/2018.5.JNS18153.

* J.M.P. and R.P. contributed equally to this work. 

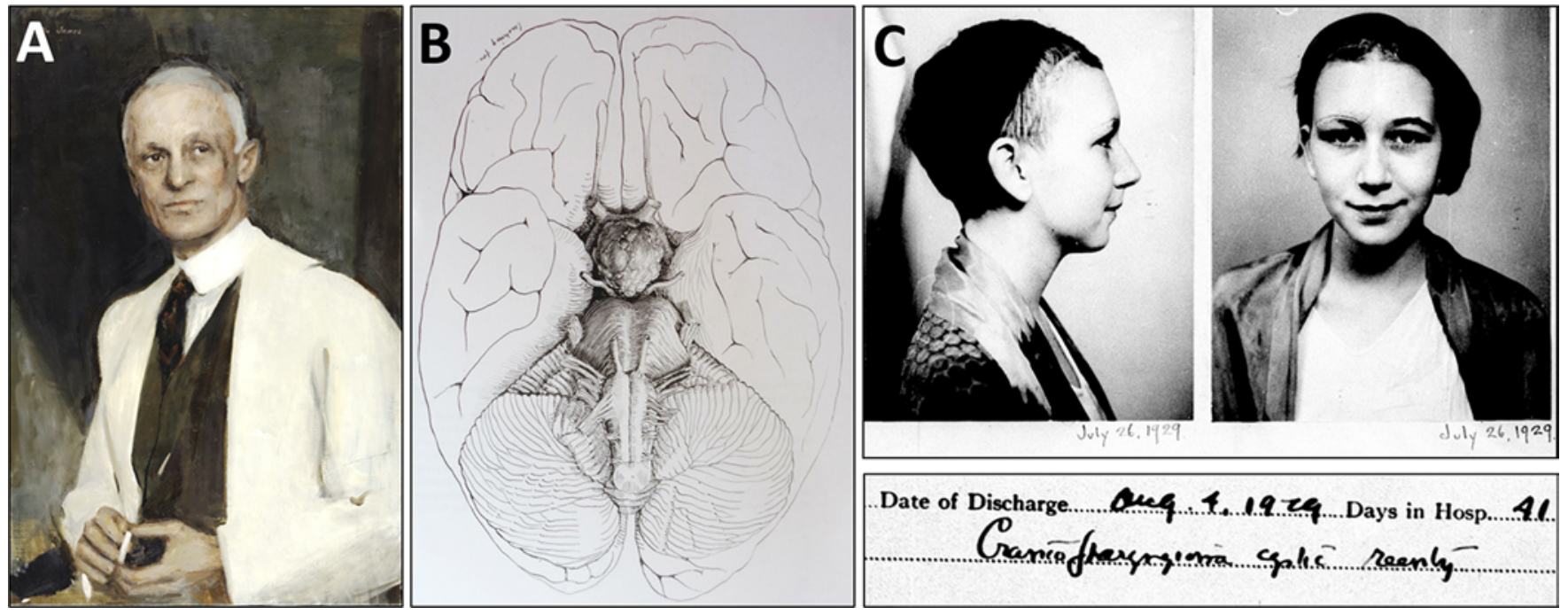

FIG. 1. A: Portrait of Harvey Williams Cushing (1869-1939), artist William James, 19th-20th century, oil on canvas, sight: 75.7 $\times 50.4 \mathrm{~cm}$, framed: $90.5 \times 65.4 \times 3.8 \mathrm{~cm}$, Harvard University Portrait Collection, gift of the three daughters of Harvey Williams Cushing to the Medical School, 1948, HNA72. It is hung at Harvard's Countway Library of Medicine, Boston, Massachusetts. Photograph Imaging Department (C) President and Fellows of Harvard College. Reprinted with the kind permission of the Harvard Art Museums. B: Drawing of an interpeduncular cyst, sketched by Harvey Cushing at the time of autopsy. It corresponds to the first intracranial tumor (case 1) surgically treated by Cushing, February 1902, at Johns Hopkins Hospital. Although this lesion was considered a "teratoma" by William Welch (1850-1934), head of the Hopkins Pathology Department at the time, multiple sources of evidence indicate that this tumor actually corresponds to a CP. From Cushing: J Nerv Ment Dis 33:704-716, 1906. Public domain. C: Pictures of a patient (case 110) taken on July 26, 1929, and a discharge note showing Cushing's handwritten word "craniopharyngioma," its first appearance in his BTR. From Cushing's BTR. Public domain. Panels B and C courtesy of the Manuscripts and Archives department of the Sterling Memorial Library, Yale University. Figure is available in color online only.

pituitary adenomas, acoustic schwannomas, gliomas, and meningiomas. ${ }^{5,14,17,19}$ Above all these tumors, however, was a seemingly benign type of suprasellar lesion that puzzled Cushing and over which he obsessed throughout his career, as it very often proved intractable (Fig. 1B). ${ }^{31}$ Cushing himself coined the term by which this tumor type is universally known today: "craniopharyngioma" (CP; Fig. 1C).27,34

Cushing failed to publish a complete, in-depth review of his pioneering 30-year surgical experience with CPs, the first large series of these complex tumors in the history of medicine. This is particularly regrettable as there are many valuable lessons to be learned from his observations on CPs, which remain a major neurosurgical challenge. From his statements, we know that he aimed to publish his series as, in 1929, he had commissioned the task of reviewing all of his material to his Dutch trainee, Ignace Oljenick..$^{9,27}$ In the end, Oljenick was unable to accomplish the project. For this reason, Cushing only prepared a brief report on his extensive, unparalleled surgical knowledge of CPs in a chapter included in his monograph Intracranial Tumors, published in 1932, the same year as his retirement. ${ }^{11}$ A complete account of Cushing's CP series has never been produced.

Providentially, Harvey Cushing painstakingly recorded, documented, and maintained every patient's history, including the tumor tissue and brain specimens obtained from his operations and autopsies. This priceless collection, known as the Cushing Brain Tumor Registry (BTR), is preserved at Yale University. Thus, a systematic, in-depth analysis of his work can be performed. In this study, we present a comprehensive analysis of Harvey Cushing's entire CP series, as documented in the records of the BTR. For a proper assessment of this material, we divided our study into two complementary parts: Part 1 analyzes how Cushing's conception of CPs evolved and the way his clinical and pathological methodology led him to achieve accurate diagnoses, and Part 2 addresses in detail Cushing's surgical strategies and the factors influencing patient outcome.

\section{Methods}

\section{Database Generation and Case Selection Criteria}

We systematically examined a microfilmed copy of the Cushing BTR stored in the Manuscripts and Archives department at Sterling Memorial Library, Yale University, New Haven, Connecticut (Harvey Cushing Papers, boxes 89-107, http://hdl.handle.net/10079/fa/mssa.ms.0160). We retrieved all the clinical charts of the tumors whose diagnosis was compatible with $\mathrm{CP}$, plus two additional cases not identified within the BTR but reported in one of Cushing's papers and in a book reviewing Cushing's work. ${ }^{8,10}$

\section{Craniopharyngioma Subcohorts Considered}

Cushing's series comprises 124 patients with a diagnosis compatible with $\mathrm{CP}\left(\mathrm{CP}_{124}\right.$; Table 1$)$. Two subcohorts within this collection are differentiated: 1) a subcohort of pathologically verified $\mathrm{CPs}\left(\mathrm{CP}_{92} ; \mathrm{n}=92\right)$, which comprises the patients whose diagnosis was confirmed by Cushing through postmortem examination, histological examination, and/or gross surgical features; and 2) a subcohort of nonverified CPs $(n=32)$, which comprises the patients 
TABLE 1. Harvey Cushing's surgically treated CP series: summary of epidemiological, clinical, pathological, and diagnostic data

\begin{tabular}{|c|c|c|c|c|c|c|c|c|c|}
\hline $\begin{array}{l}\text { Case } \\
\text { No. }\end{array}$ & $\begin{array}{l}\text { Patient Initials, } \\
\text { Yr of Presentation } \\
\text { (surgical ID) }\end{array}$ & $\begin{array}{c}\text { Age } \\
\text { (yrs), } \\
\text { Sex }\end{array}$ & $\begin{array}{c}\text { Time } \\
\text { Course } \\
\text { up to Dx }\end{array}$ & Clinical Symptoms & $\begin{array}{c}\text { Major } \\
\text { Syndrome }\end{array}$ & Rx Signs & $\begin{array}{c}\text { Diagnostic } \\
\text { Evidence/Hist }\end{array}$ & Topography & $\begin{array}{c}\text { Pathology } \\
\text { Verified }\end{array}$ \\
\hline 1 & M.D., 1901 (13054) & $16, \mathrm{~F}$ & 3 yrs & $\mathrm{H}, \mathrm{V}, \mathrm{Fr}$, Som, B, T, C & ITS & Not done & Necr/teratoma & IT & No \\
\hline 2 & A.B.W., 1908 (22665) & $56, F$ & 14 yrs & H, V, E, Fr, DI, Som, T, A & ITS & SS calcif & Rx/NA & IT & No \\
\hline 3 & Unknown, 1909 (NA) & $36, F$ & $7 \mathrm{mos}$ & $\mathrm{H}, \mathrm{V}, \mathrm{E}, \mathrm{Fr}, \mathrm{DI}$ & ITS & S normal & Clin/NA & IT & No \\
\hline 4 & L.T., 1910 (25715) & $15, F$ & $10 \mathrm{yrs}$ & H, V, E, Fr, DI, T, Szs & ITS & S enlarged & Clin, Rx/NA & S/SS-3V & No \\
\hline 5 & E.D., 1910 (26920) & $18, F$ & $10 \mathrm{yrs}$ & H, E, Cx, DI, Som, B, T, C & Hyp & S eroded & Clin, Rx/NA & IT & No \\
\hline 6 & D.M.B., 1911 (28747) & $31, \mathrm{M}$ & $4 \mathrm{yrs}$ & $\mathrm{H}, \mathrm{V}, \mathrm{E}, \mathrm{Som}, \mathrm{B}, \mathrm{Me}$ & Hyp & S normal & Necr/papillary & Strict 3V & Yes \\
\hline 7 & W.B.T., 1911 (28818) & $48, M$ & $5 \mathrm{yrs}$ & H, V, E, DI, Som, B, Me, C & Hyp & S normal & Necr/papillary & IT & Yes \\
\hline 8 & I.B.A., 1911 (29150) & $33, \mathrm{M}$ & 3 yrs & $\mathrm{H}, \mathrm{V}$ & Chi & S eroded & Necr/adam & S/SS-3V & Yes \\
\hline 9 & J.C.A., 1913 (18911) & $20, \mathrm{M}$ & $7 \mathrm{yrs}$ & $\mathrm{H}, \mathrm{V}, \mathrm{E}$ & Chi-Pi & S calcif & Hist/adam & S/SS & Yes \\
\hline 10 & A.L.R., 1913 (41) & $25, F$ & $2 \mathrm{yrs}$ & $\mathrm{H}, \mathrm{V}, \mathrm{E}, \mathrm{B}, \mathrm{Me}, \mathrm{Szs}$ & Hyp & SS calcif & Rx, AirV/NA & S/SS-3V & No \\
\hline 11 & K.L., 1913 (663) & $11, \mathrm{~F}$ & $6 \mathrm{yrs}$ & $\mathrm{H}, \mathrm{V}, \mathrm{E}, \mathrm{B}$ & Chi & SS calcif & Hist/teratoid & Pseudo 3V & Yes \\
\hline 12 & O.A.F., 1913 (13-178) & $20, \mathrm{M}$ & $18 \mathrm{yrs}$ & H, V, E, Som, Szs & $\mathrm{HICP}$ & S eroded & Necr/adam & Pseudo 3V & Yes \\
\hline 13 & M.N.M., 1913 (75) & $58, F$ & $1 \mathrm{yr}$ & V, O, DI & Chi & S enlarged & Clin/NA & NA & No \\
\hline 14 & J.C.N., 1913 (22-A) & $51, \mathrm{~F}$ & $1 \mathrm{yr}$ & $\mathrm{H}, \mathrm{V}, \mathrm{Fr}, \mathrm{DI}$ & Chi-ITS & S eroded & Clin, Rx/NA & NA & No \\
\hline 15 & H.W.F., 1913 (164) & $12, \mathrm{M}$ & $1 \mathrm{yr}$ & $\mathrm{H}, \mathrm{V}, \mathrm{E}, \mathrm{O}$ & Chi-Pi & S eroded & Clin, Rx/NA & NA & No \\
\hline 16 & A.F.B., 1913 (209) & $28, F$ & $15 \mathrm{yrs}$ & V, E, Fr, DI, Som & Chi-Pi & SS calcif & Clin, Rx /NA & S/SS-3V & No \\
\hline 17 & D.A., 1913 (235) & $14, \mathrm{~F}$ & $7 \mathrm{yrs}$ & H, V, Fr, Cx, DI, Som, B, C & All5 & SS calcif & Hist/teratoid & S/SS-3V & Yes \\
\hline 18 & L.E.M., 1913 (303) & $12, \mathrm{M}$ & $2 \mathrm{yrs}$ & $\mathrm{H}, \mathrm{DI}$, Som, B, Me, T, A, G & Hyp & S normal & Clin/NA & S/SS-3V & No \\
\hline 19 & E.S., 1913 (1009) & $19, \mathrm{~F}$ & 13 yrs & $\mathrm{H}, \mathrm{V}, \mathrm{Fr}$ & $\mathrm{HICP}$ & S normal & Clin/NA & IT & No \\
\hline 20 & B.A., 1913 (538) & $51, \mathrm{~F}$ & 3 yrs & H, V, B, Me, Szs, CN VI palsy & $\mathrm{HICP}$ & S eroded & Hist/teratoid & S/SS-3V & Yes \\
\hline 21 & A.D.S., 1914 (1036) & $41, \mathrm{~F}$ & $6 \mathrm{mos}$ & $\mathrm{H}, \mathrm{V}, \mathrm{Fr}$, Som, B, Me & Hyp & S eroded & Necr/teratoid & IT & Yes \\
\hline 22 & S.D., 1914 (1033) & $30, F$ & $15 \mathrm{yrs}$ & $H, V, E, B$ & Chi-Pi & S/SS calcif & Hist/epith cyst & S/SS & Yes \\
\hline 23 & H.B., 1914 (1291) & $19, \mathrm{~F}$ & $7 \mathrm{yrs}$ & $\mathrm{H}, \mathrm{E}, \mathrm{Fr}$, Som, B, Me & ITS-Hyp & S eroded & Clin, Rx/NA & NA & No \\
\hline 24 & E.M.S., 1914 (1440) & $49, \mathrm{~F}$ & $10 \mathrm{yrs}$ & H, V, Fr, Som, B, Me, G & All5 & SS calcif & Clin, Rx/NA & NA & No \\
\hline 25 & O.M.C., 1914 (1583) & $23, \mathrm{~F}$ & $2 \mathrm{yrs}$ & $\mathrm{H}, \mathrm{V}, \mathrm{E}, \mathrm{CN}$ VII palsy & HICP & S normal & Clin/pit cyst & NA & No \\
\hline 26 & L.J.B., 1914 (1629) & $27, \mathrm{~F}$ & $1 \mathrm{yr}$ & $H, V, E$, Szs & Chi-Pi & S enlarged & Surg/pit cyst & Pseudo 3V & Yes \\
\hline 27 & B.S.L., 1914 (2114) & $40, F$ & $20 \mathrm{yrs}$ & $\mathrm{H}, \mathrm{V}, \mathrm{Fr}, \mathrm{DI}, \mathrm{Som}, \mathrm{B}, \mathrm{Me}, \mathrm{G}$ & ITS-Hyp & S calcif & Surg/S teeth & S/SS-3V & No \\
\hline 28 & D.C., 1914 (2186) & $48, M$ & $2.5 \mathrm{yrs}$ & $\mathrm{H}, \mathrm{V}, \mathrm{E}, \mathrm{DI}$ & Chi-Pi & S enlarged & Hist/pit cyst & S/SS & No \\
\hline 29 & A.L.B., 1914 (2153) & $11, \mathrm{~F}$ & $1 \mathrm{yr}$ & $\mathrm{H}, \mathrm{V}, \mathrm{E}, \mathrm{DI}$ & $\mathrm{HICP}$ & SS calcif & Necr/SS cyst & IT & Yes \\
\hline 30 & C.K., 1914 (2268) & $21, M$ & 3 yrs & H, V, B, Me, G, Ul, C, Szs & Hyp & S enlarged & Clin, Rx/NA & S/SS-3V & No \\
\hline 31 & H.J.B., 1915 (2323) & $15, M$ & 3 yrs & V, E, Fr, DI, Som, B, Szs & ITS & S enlarged & Clin, Rx/NA & IT & No \\
\hline 32 & T.L.D., 1915 (3185) & $20, M$ & $6 \mathrm{mos}$ & H, V, E, Som & Chi-Pi & S enlarged & Hist/cholest & S/SS-3V & Yes \\
\hline 33 & H.B., 1915 (3572) & $30, \mathrm{M}$ & NA & $\mathrm{H}, \mathrm{V}, \mathrm{E}, \mathrm{Som}$ & Chi-Pi & S enlarged & Hist/adam & S/SS & Yes \\
\hline 34 & Unknown, 1915 (3883) & $30, F$ & NA & H, V, Fr, Som, Szs & $\mathrm{HICP}$ & S enlarged & Surg/pit cyst & S/SS-3V & No \\
\hline 35 & M.C.L., 1916 (4306) & $23, \mathrm{~F}$ & $1.5 \mathrm{yrs}$ & $H, V, E$, Szs & $\mathrm{HICP}$ & S eroded & Clin, Rx/NA & S/SS-3V & No \\
\hline 36 & M.F.W., 1916 (4487) & $31, \mathrm{~F}$ & $10 \mathrm{yrs}$ & $\mathrm{H}, \mathrm{V}, \mathrm{E}, \mathrm{Fr}$ & Chi-Pi & S eroded & Surg/SS cyst & S/SS & No \\
\hline 37 & E.C., 1916 (5617) & $8, F$ & $3 \mathrm{mos}$ & $\mathrm{H}, \mathrm{V}, \mathrm{B}, \mathrm{T}, \mathrm{UI}, \mathrm{C}$ & $\mathrm{HICP}$ & S eroded & Clin, Rx/NA & NA & No \\
\hline 38 & M.H.D., 1916 (5839) & $20, F$ & $5 \mathrm{yrs}$ & $\mathrm{H}, \mathrm{V}, \mathrm{DI}, \mathrm{G}$ & $\mathrm{HICP}$ & SS calcif & Surg/SS cyst & Pseudo 3V & Yes \\
\hline 39 & L.S., 1917 (6615) & $16, \mathrm{~F}$ & 3 yrs & H, V, E, Fr, DI, Som & $\mathrm{HICP}$ & SS calcif & Surg/SS cyst & S/SS-3V & Yes \\
\hline 40 & R.I.R., 1919 (11152) & $13, \mathrm{M}$ & $6 \mathrm{yrs}$ & $H, V, E$ & Chi-Pi & S eroded & Surg/cholest & Pseudo 3V & No \\
\hline 41 & L.W., 1919 (10067) & $15, \mathrm{~F}$ & $2 \mathrm{yrs}$ & H, V, E, DI, Som, B, Me, T, G & All5 & SS calcif & Hist/dermoid & S/SS-3V & Yes \\
\hline 42 & J.P., 1919 (10563) & $14, \mathrm{M}$ & 4 yrs & H, V, E, Som, B, G & $\mathrm{HICP}$ & SS calcif & Necr/adam & IT & Yes \\
\hline 43 & F.E., 1919 (10779) & $58, F$ & $4 \mathrm{yrs}$ & $\mathrm{H}, \mathrm{V}, \mathrm{E}, \mathrm{O}$ & $\mathrm{HICP}$ & S enlarged & Hist/cholest & S/SS & Yes \\
\hline 44 & W.C.C., 1919 (11306) & $34, \mathrm{M}$ & $17 \mathrm{yrs}$ & H, V, Fr, DI, B & ITS & S/SS calcif & Hist/adam & Pseudo 3V & Yes \\
\hline 45 & J.A.P., 1919 (11541) & $17, \mathrm{M}$ & $15 \mathrm{yrs}$ & H, V, E, DI, Som, CN III palsy & Chi-Pi & S/SS calcif & Hist/adam & S/SS & Yes \\
\hline 46 & J.A.L., 1920 (11764) & $30, F$ & $15 \mathrm{yrs}$ & H, V, DI, Som, B & $\mathrm{HICP}$ & SS calcif & Surg/SS cyst & S/SS-3V & Yes \\
\hline 47 & C.L.A., 1920 (12292) & $35, \mathrm{~F}$ & $2 \mathrm{yrs}$ & H, V, E, DI, Som, B, Me & ITS & S normal & Hist/papillary & IT & Yes \\
\hline 48 & M.M.R., 1920 (12502) & $18, F$ & $2 \mathrm{yrs}$ & $V, E$ & Chi-Pi & S enlarged & Surg/SS cyst & S/SS & Yes \\
\hline
\end{tabular}


" CONTINUED FROM PAGE 951

TABLE 1. Harvey Cushing's surgically treated CP series: summary of epidemiological, clinical, pathological, and diagnostic data

\begin{tabular}{|c|c|c|c|c|c|c|c|c|c|}
\hline $\begin{array}{l}\text { Case } \\
\text { No. }\end{array}$ & $\begin{array}{l}\text { Patient Initials, } \\
\text { Yr of Presentation } \\
\text { (surgical ID) }\end{array}$ & $\begin{array}{l}\text { Age } \\
\text { (yrs), } \\
\text { Sex }\end{array}$ & $\begin{array}{c}\text { Time } \\
\text { Course } \\
\text { up to Dx }\end{array}$ & Clinical Symptoms & $\begin{array}{c}\text { Major } \\
\text { Syndrome }\end{array}$ & Rx Signs & $\begin{array}{c}\text { Diagnostic } \\
\text { Evidence/Hist }\end{array}$ & Topography & $\begin{array}{l}\text { Pathology } \\
\text { Verified }\end{array}$ \\
\hline 49 & J.J.C., 1920 (13110) & $12, \mathrm{M}$ & $1 \mathrm{yr}$ & H, V, Dip & Chi & S enlarged & Surg/SS cyst & S/SS-3V & No \\
\hline 50 & S.S.N., 1920 (13453) & $33, \mathrm{M}$ & 2 yrs & $H, V, E$ & Chi-Pi & S eroded & Hist/papillary & Pseudo 3V & Yes \\
\hline 51 & B.B., 1920 (13553) & $25, F$ & $2 \mathrm{yrs}$ & $\mathrm{H}, \mathrm{V}, \mathrm{Fr}$ & $\mathrm{HICP}$ & S eroded & Hist/papillary & Pseudo 3V & No \\
\hline 52 & F.S.D., 1921 (35075) & $34, \mathrm{M}$ & $1.5 \mathrm{yrs}$ & $\mathrm{H}, \mathrm{V}, \mathrm{E}, \mathrm{Fr}$, Som, B & $\mathrm{HICP}$ & SS calcif & Necr/adam & S/SS-3V & Yes \\
\hline 53 & H.M.S., 1921 (14011) & $11, \mathrm{M}$ & $5 \mathrm{yrs}$ & $\mathrm{H}, \mathrm{V}, \mathrm{E}, \mathrm{O}$ & Chi & S enlarged & Necr/adam & Pseudo 3V & Yes \\
\hline 54 & T.D.L., 1921 (14327) & $45, \mathrm{~F}$ & 30 yrs & $\mathrm{H}, \mathrm{V}, \mathrm{Fr}, \mathrm{DI}, \mathrm{Som}, \mathrm{B}, \mathrm{Me}, \mathrm{T}, \mathrm{A}$ & All5 & SS calcif & Necr/adam & S/SS-3V & Yes \\
\hline 55 & E.P.W., 1921 (14817) & $31, F$ & $5 \mathrm{yrs}$ & V, Fr, Som & Chi-ITS & S normal & Necr/adam & IT & Yes \\
\hline 56 & M.H.T., 1921 (14996) & $57, \mathrm{~F}$ & $6 \mathrm{yrs}$ & V, DI, O & Chi-ITS & S normal & Hist/papillary & IT & Yes \\
\hline 57 & D.E.H., 1921 (17015) & $6, F$ & 3 yrs & $\mathrm{H}, \mathrm{V}, \mathrm{O}, \mathrm{Szs}$ & $\mathrm{HICP}$ & SS calcif & Necr/adam & IT & Yes \\
\hline 58 & J.L.K., 1922 (16147) & $15, \mathrm{M}$ & $2 \mathrm{yrs}$ & $\mathrm{H}, \mathrm{V}, \mathrm{Fr}$, Som, Me, A, Szs & Chi-ITS & SS calcif & Hist/adam & S/SS-3V & Yes \\
\hline 59 & G.H.A., 1922 (16889) & $21, \mathrm{M}$ & $7 \mathrm{yrs}$ & H, V, Fr, Som, B, Szs & ITS & SS calcif & Necr/adam & S/SS-3V & Yes \\
\hline 60 & C.B.H., 1922 (17148) & $23, \mathrm{M}$ & $15 \mathrm{yrs}$ & $H, V, E$ & Chi-Pi & SS calcif & Hist/adam & S/SS-3V & Yes \\
\hline 61 & C.B.M., 1922 (17444) & $21, \mathrm{M}$ & $7 \mathrm{yrs}$ & $\begin{array}{l}\text { H, V, E, Som, Me, Szs, CN } \\
\quad \text { III palsy }\end{array}$ & $\mathrm{HICP}$ & S eroded & Clin, Rx/NA & S/SS-3V & No \\
\hline 62 & M.E.K., 1922 (17650) & $10, F$ & $6 \mathrm{yrs}$ & $\mathrm{H}, \mathrm{V}, \mathrm{E}, \mathrm{Cx}$ & Chi-Pi & SS calcif & Hist/adam & Pseudo 3V & Yes \\
\hline 63 & B.A., 1922 (17754) & $40, M$ & $5 \mathrm{yrs}$ & $\mathrm{H}, \mathrm{V}, \mathrm{E}, \mathrm{B}$, It paresis & Chi & S normal & Hist/cholest & Pseudo 3V & No \\
\hline 64 & J.A.D., 1922 (17844) & $12, \mathrm{M}$ & 6 yrs & $\mathrm{V}, \mathrm{E}$, leg paresis & Pi-Chi & SS calcif & Hist/adam & S/SS & Yes \\
\hline 65 & A.V.F., 1922 (17876) & $13, F$ & $7 \mathrm{yrs}$ & $\mathrm{H}, \mathrm{V}, \mathrm{E}, \mathrm{B}$ & $\mathrm{HICP}$ & SS calcif & Hist/adam & S/SS-3V & Yes \\
\hline 66 & P.S., 1922 (19274) & $15, M$ & $2 \mathrm{yrs}$ & H, V, Fr, Som, Szs & Chi-ITS & SS calcif & Necr/adam & S/SS-3V & Yes \\
\hline 67 & W.C.C., 1923 (18658) & $25, M$ & $10 \mathrm{yrs}$ & $\mathrm{H}, \mathrm{V}, \mathrm{E}, \mathrm{Cx}$ & Pi-Chi & SS calcif & Hist/adam & S/SS & Yes \\
\hline 68 & H.V.M., 1923 (18686) & $6, M$ & $2.5 \mathrm{yrs}$ & H, V, Szs & Chi & SS calcif & Hist/adam & S/SS-3V & Yes \\
\hline 69 & J.R., 1923 (18815) & $19, F$ & $5 \mathrm{yrs}$ & $\mathrm{H}, \mathrm{V}, \mathrm{E}, \mathrm{DI}$ & $\mathrm{HICP}$ & SS calcif & Hist/adam & Pseudo 3V & Yes \\
\hline 70 & F.W.V., 1923 (18827) & $43, M$ & $1.5 \mathrm{yrs}$ & V, H, E, Som, B, Me, G, UI & Hyp & S normal & Hist/papillary & Strict 3V & Yes \\
\hline 71 & L.M.K., 1923 (20045) & $57, \mathrm{~F}$ & $6 \mathrm{mos}$ & $\mathrm{H}, \mathrm{V}, \mathrm{O}$ & Chi & S enlarged & Surg/adam & S/SS & Yes \\
\hline 72 & M.P., 1923 (20411) & $18, F$ & $2 \mathrm{mos}$ & $\mathrm{H}, \mathrm{V}, \mathrm{E}, \mathrm{G}$ & $\mathrm{HICP}$ & SS calcif & Hist/adam & Pseudo 3V & Yes \\
\hline 73 & K.K.S., 1924 (22493) & $19, \mathrm{M}$ & $10 \mathrm{yrs}$ & $\mathrm{H}, \mathrm{E}, \mathrm{Fr}, \mathrm{DI}$ & Pi-ITS & SS calcif & Hist/adam & S/SS & Yes \\
\hline 74 & T.H.C., 1924 (20737) & $5, M$ & $4 \mathrm{yrs}$ & $\mathrm{H}, \mathrm{V}, \mathrm{Cx}$, Som, C & Chi & S calcif & Necr/adam & S/SS-3V & Yes \\
\hline 75 & A.M., 1924 (20814) & $20, F$ & $4 \mathrm{yrs}$ & $\mathrm{H}, \mathrm{V}, \mathrm{Fr}$, Som, B, Me, C & ITS-Hyp & SS calcif & Hist/mixed & IT & Yes \\
\hline 76 & A.G.S., 1924 (22004) & $38, \mathrm{M}$ & $20 \mathrm{yrs}$ & V, Fr, DI, B, Me, T, G, C & ITS-Hyp & SS calcif & Necr/adam & IT & Yes \\
\hline 77 & J.C., 1925 (22999) & $10, M$ & 5 yrs & $\mathrm{H}, \mathrm{V}, \mathrm{E}$ & $\mathrm{HICP}$ & SS calcif & Hist/adam & S/SS & Yes \\
\hline 78 & L.G.B., 1925 (24292) & $51, \mathrm{M}$ & 3 yrs & $\mathrm{H}, \mathrm{V}, \mathrm{E}, \mathrm{Som}, \mathrm{B}, \mathrm{Me}$ & Hyp & SS calcif & Necr/adam & Strict 3V & Yes \\
\hline 79 & M.T.S., 1925 (31011) & $62, \mathrm{~F}$ & $1 \mathrm{yr}$ & $\mathrm{H}, \mathrm{V}$ & Chi & S normal & Hist/adam & S/SS-3V & Yes \\
\hline 80 & L.T., 1925 (28813) & $3, F$ & $6 \mathrm{mos}$ & H, V, E, Cx, DI, Som, B & All5 & SS calcif & Necr/adam & Pseudo 3V & Yes \\
\hline 81 & H.F.M., 1925 (25383) & $26, M$ & $2.5 \mathrm{yrs}$ & $H, V, E$ & Chi & S eroded & Necr/adam & S/SS-3V & Yes \\
\hline 82 & M.J.R., 1926 (28247) & $6, F$ & $1 \mathrm{yr}$ & $\begin{array}{l}\mathrm{H}, \mathrm{V}, \mathrm{E}, \mathrm{Fr}, \mathrm{DI}, \text { Som, CN VI } \\
\text { palsy }\end{array}$ & $\mathrm{HICP}$ & SS calcif & Hist/adam & IT & Yes \\
\hline 83 & H.L.R., 1926 (25714) & $12, \mathrm{M}$ & 6 yrs & H, V, Fr, DI, C & $\mathrm{HICP}$ & SS calcif & Hist/adam & S/SS-3V & Yes \\
\hline 84 & H.W., 1926 (25886) & $17, \mathrm{M}$ & $4 \mathrm{yrs}$ & V, E, Fr, DI, Som & Chi-Pi & S/SS calcif & Hist/adam & S/SS-3V & Yes \\
\hline 85 & E.H., 1926 (27320) & $8, M$ & $6 \mathrm{mos}$ & $\mathrm{H}, \mathrm{V}, \mathrm{E}, \mathrm{Som}, \mathrm{B}, \mathrm{Me}, \mathrm{G}, \mathrm{C}$ & $\mathrm{HICP}$ & SS calcif & Rx/NA & S/SS-3V & No \\
\hline 86 & A.C.K., 1926 (26734) & $25, M$ & $15 \mathrm{yrs}$ & $\mathrm{H}, \mathrm{V}, \mathrm{E}, \mathrm{Fr}$ & ITS-Chi & SS calcif & Hist/adam & Pseudo 3V & Yes \\
\hline 87 & J.J.S., 1926 (26200) & $10, M$ & $5.5 \mathrm{yrs}$ & H, V, E, DI & Chi-Pi & SS calcif & Hist/CP & S/SS-3V & Yes \\
\hline 88 & A.F.M., 1926 (26287) & $23, \mathrm{M}$ & $14 \mathrm{yrs}$ & H, V, E, DI, B, progeria & Chi-Pi & SS calcif & Hist/adam & S/SS-3V & Yes \\
\hline 89 & H.M.S., 1926 (26470) & $62, \mathrm{~F}$ & $9 \mathrm{mos}$ & $\mathrm{H}, \mathrm{V}$ & Chi & S normal & Hist/papillary & Pseudo 3V & Yes \\
\hline 90 & L.V., 1926 (26753) & $36, M$ & $15 \mathrm{yrs}$ & H, V, E, DI & Pi-Chi & SS calcif & Hist/adam & Pseudo 3V & Yes \\
\hline 91 & F.W., 1926 (27041) & $27, \mathrm{M}$ & $7 \mathrm{yrs}$ & $\mathrm{H}, \mathrm{V}, \mathrm{Fr}, \mathrm{DI}, \mathrm{B}, \mathrm{Me}, \mathrm{G}, \mathrm{C}$ & All5 & SS calcif & Hist/adam & IT & Yes \\
\hline 92 & J.A.B., 1926 (27398) & $30, F$ & $15 \mathrm{yrs}$ & H, E, DI, Som, B, Me, G, C & All5 & SS calcif & Necr/adam & S/SS-3V & Yes \\
\hline 93 & A.Z., 1927 (33831) & $32, \mathrm{M}$ & $6 \mathrm{mos}$ & H, DI, Som, B, Me, G, C, Szs & Hyp & SS calcif & Hist/adam & IT & Yes \\
\hline
\end{tabular}


TABLE 1. Harvey Cushing's surgically treated CP series: summary of epidemiological, clinical, pathological, and diagnostic data

\begin{tabular}{|c|c|c|c|c|c|c|c|c|c|}
\hline $\begin{array}{l}\text { Case } \\
\text { No. }\end{array}$ & $\begin{array}{l}\text { Patient Initials, } \\
\text { Yr of Presentation } \\
\text { (surgical ID) }\end{array}$ & $\begin{array}{c}\text { Age } \\
\text { (yrs), } \\
\text { Sex }\end{array}$ & $\begin{array}{c}\text { Time } \\
\text { Course } \\
\text { up to Dx }\end{array}$ & Clinical Symptoms & $\begin{array}{c}\text { Major } \\
\text { Syndrome }\end{array}$ & Rx Signs & $\begin{array}{c}\text { Diagnostic } \\
\text { Evidence/Hist }\end{array}$ & Topography & $\begin{array}{l}\text { Pathology } \\
\text { Verified }\end{array}$ \\
\hline 94 & M.E.D., 1927 (28383) & $8, F$ & $2 \mathrm{yrs}$ & $\mathrm{H}, \mathrm{V}, \mathrm{E}, \mathrm{B}, \mathrm{Me}$ & $\mathrm{HICP}$ & SS calcif & Necr/adam & IT & Yes \\
\hline 95 & H.L., 1927 (30302) & $13, \mathrm{M}$ & $2 \mathrm{mos}$ & $\mathrm{H}, \mathrm{V}, \mathrm{E}, \mathrm{DI}, \mathrm{Som}, \mathrm{B}, \mathrm{Me}$ & All5 & SS calcif & Hist/adam & S/SS-3V & Yes \\
\hline 96 & E.J., 1927 (29467) & $6, M$ & 4 yrs & $\mathrm{H}, \mathrm{V}, \mathrm{E}$, It paresis & $\mathrm{HICP}$ & SS calcif & Hist/adam & $\begin{array}{l}\text { All intracra- } \\
\text { nial spaces } \\
\text { (giant) }\end{array}$ & Yes \\
\hline 97 & R.L.J., 1927 (31011) & $6, F$ & $2 \mathrm{yrs}$ & H, V, E, DI, Som, B, G, Szs & All5 & SS calcif & Hist/adam & S/SS-3V & Yes \\
\hline 98 & J.L.B., 1927 (32940) & $6, \mathrm{M}$ & $1 \mathrm{yr}$ & H, V, DI, Som, B, Szs & $\mathrm{HICP}$ & SS calcif & Hist/RC-CP & IT & Yes \\
\hline 99 & H.O.C., 1927 (32718) & $24, F$ & $5 \mathrm{yrs}$ & H, V, Som, B, Szs & $\mathrm{HICP}$ & $3 \mathrm{~V}$ calcif & $\mathrm{Rx} / \mathrm{NA}$ & Strict 3V & No \\
\hline 100 & E.I.G., 1928 (31094) & $20, F$ & $8 \mathrm{yrs}$ & H, V, E, DI, B & Pi-Chi & S eroded & Surg/CP & S/SS & Yes \\
\hline 101 & N.C.S., 1928 (31467) & $10, F$ & $1.5 \mathrm{yrs}$ & V, Fr, Som, Szs & $\mathrm{HICP}$ & SS calcif & Hist/adam & S/SS-3V & Yes \\
\hline 102 & N.F.S., 1928 (31979) & $50, F$ & $5 \mathrm{yrs}$ & $\mathrm{H}, \mathrm{V}, \mathrm{Fr}$, Som, UI & Chi-ITS & SS calcif & Hist/adam & S/SS-3V & Yes \\
\hline 103 & M.V.O., 1928 (32294) & $10, F$ & $7 \mathrm{yrs}$ & $\mathrm{H}, \mathrm{V}, \mathrm{E}, \mathrm{O}, \mathrm{Som}, \mathrm{T}, \mathrm{G}$ & $\mathrm{HICP}$ & SS calcif & Rx/NA & S/SS-3V & No \\
\hline 104 & A.B., 1928 (32298) & $22, \mathrm{~F}$ & $10 \mathrm{yrs}$ & H, V, Fr, DI, Som, B, UI & Chi-ITS & S eroded & Hist/teratoid & S/SS-3V & Yes \\
\hline 105 & K.K.S., 1928 (32358) & $46, M$ & $6 \mathrm{mos}$ & V, Fr, DI, Som, B, Me, Ul & ITS-Hyp & S normal & Necr/adam & IT & Yes \\
\hline 106 & L.R.C., 1928 (32590) & $40, F$ & $1 \mathrm{yr}$ & $\mathrm{H}, \mathrm{V}$ & Chi & SS calcif & Hist/adam & Pseudo 3V & Yes \\
\hline 107 & S.B., 1928 (32718) & $30, \mathrm{M}$ & $1 \mathrm{yr}$ & $\mathrm{H}, \mathrm{V}, \mathrm{E}, \mathrm{DI}$ & Pi-Chi & S eroded & Surg/CP & S/SS & Yes \\
\hline 108 & M.A.L., 1928 (32851) & $9, M$ & 3 yrs & $\mathrm{H}, \mathrm{V}, \mathrm{Fr}$ & Chi & SS calcif & Hist/adam & Pseudo 3V & Yes \\
\hline 109 & M.V.M., 1929 (33044) & $19, \mathrm{~F}$ & $1 \mathrm{mo}$ & $\mathrm{H}, \mathrm{V}, \mathrm{Fr}$ & $\mathrm{HICP}$ & SS calcif & Hist/adam & IT & Yes \\
\hline 110 & B.L.E., 1929 (34286) & $15, F$ & $2 \mathrm{yrs}$ & $\mathrm{H}, \mathrm{V}, \mathrm{E}, \mathrm{DI}$ & Chi-Pi & S calcif & Hist/adam & S/SS & Yes \\
\hline 111 & M.Н., 1929 (35047) & $43, F$ & $2 \mathrm{mos}$ & H, V, E, O, Som, B, T, UI, C & $\mathrm{HICP}$ & SS calcif & Necr/adam & IT & Yes \\
\hline 112 & N.A.K., 1930 (35581) & $16, \mathrm{M}$ & $6 \mathrm{mos}$ & $\mathrm{V}, \mathrm{E}$ & Chi & S enlarged & Hist/adam & S/SS & Yes \\
\hline 113 & E.M.L., 1930 (35994) & $27, \mathrm{~F}$ & 10 mos & H, V, E, B, Som, Me, Szs & Hyp & SS calcif & Necr/adam & IT & Yes \\
\hline 114 & E.J., 1930 (36672) & $22, \mathrm{~F}$ & 3 yrs & H, V, Fr, DI, Som, T, G, C & ITS-Hyp & S eroded & Necr/xantho & IT & No \\
\hline 115 & M.E.P., 1930 (35969) & $29, \mathrm{M}$ & 3 yrs & H, V, Fr, DI, Som, B, Me, G & ITS-Hyp & S normal & Hist/adam & IT & Yes \\
\hline 116 & N.E.C., 1931 (38528) & $23, F$ & $10 \mathrm{yrs}$ & H, V, Fr, B, Som, Me, C, Szs & $\mathrm{HICP}$ & SS calcif & Necr/adam & IT & Yes \\
\hline 117 & S.G.W., 1931 (38556) & $6, M$ & 3 yrs & $\mathrm{H}, \mathrm{V}, \mathrm{A}, \mathrm{C}$ & $\mathrm{HICP}$ & SS calcif & Necr/adam & S/SS-3V & Yes \\
\hline 118 & C.E.O., 1931 (39201) & $70, F$ & 3 yrs & $\mathrm{H}, \mathrm{V}$ & Chi & S normal & Hist/CP-RC & S/SS & Yes \\
\hline 119 & M.B., 1931 (39270) & $21, F$ & $1 \mathrm{yr}$ & H, V, Som, G, CN VII palsy & $\mathrm{HICP}$ & S normal & Hist/CP atyp & Strict 3V & Yes \\
\hline 120 & A.C., 1932 (40686) & $51, \mathrm{~F}$ & $2 \mathrm{yrs}$ & $\mathrm{H}, \mathrm{V}$ & Chi & S normal & Hist/adam & Pseudo 3V & Yes \\
\hline 121 & M.E.T., 1932 (40786) & $40, F$ & $2 \mathrm{yrs}$ & $\mathrm{H}, \mathrm{V}, \mathrm{E}, \mathrm{O}$ & Chi & S normal & Hist/papillary & Pseudo 3V & Yes \\
\hline 122 & M.S.B., 1932 (40859) & $38, M$ & 4 yrs & H, B, O, Me, Som, G, Dip & Hyp & S normal & Hist/papillary & Strict 3V & Yes \\
\hline 123 & M.J.C., 1932 (41028) & $17, \mathrm{~F}$ & 5 yrs & $\begin{array}{l}H, V, E, G, C N \text { VII palsy, } \\
\text { deafness }\end{array}$ & HICP & SS calcif & Hist/CP odo & Pseudo 3V & Yes \\
\hline 124 & I.M.M., 1932 (41416) & $56, F$ & $2.5 \mathrm{yrs}$ & H, V, Dip & Chi & SS calcif & Hist/teratoid & Pseudo 3V & Yes \\
\hline
\end{tabular}

$\mathrm{A}=$ autonomous nervous system alterations; adam = adamantinomatous; AirV = air ventriculography; $\mathrm{All} 5=$ all 5 syndromes; atyp = atypical; $\mathrm{B}=$ behavioral disturbances; $\mathrm{C}=$ consciousness impairment; calcif = calcifications; $\mathrm{Chi}=$ chiasmatic syndrome; cholest = cholesteatoma; $\mathrm{Clin}=$ clinical; $\mathrm{CN}=\mathrm{cranial}$ nerve; $\mathrm{CP}=\mathrm{craniopharyn}-$ gioma; $\mathrm{Cx}$ = cachexia; $\mathrm{DI}$ = diabetes insipidus; $\mathrm{Dip}=$ diplopia; $\mathrm{Dx}=$ diagnosis; $\mathrm{E}=$ endocrine deficit; epith = epithelial; $\mathrm{Fr}=$ Fröhlich syndrome; $\mathrm{G}=$ gait disturbance; $\mathrm{H}=$ headache; HICP = high intracranial pressure syndrome; Hist = histology; Hyp = hypothalamic syndrome; ID = identification; IT = infundibulo-tuberal; ITS = IT syndrome; $\mathrm{Me}=$ memory defect; $\mathrm{NA}=$ not available; $\mathrm{Necr}=$ necropsy; $\mathrm{O}=$ obesity; odo = odontogenic; $\mathrm{Pi}$ = pituitary syndrome; pit = pituitary; Pseudo $3 \mathrm{~V}=$ suprasellar pseudointraventricular; RC = Rathke's cyst; Rx = skull radiograph; S = sellar; Som = somnolence; SS = suprasellar; S/SS = sellar-suprasellar; S/SS-3V = S/SS-secondary intraventricular; Strict $3 \mathrm{~V}=$ strictly intraventricular; Surg = surgery; $\mathrm{Szs}=$ seizures; $\mathrm{T}=$ body temperature alteration; teratoid $=\mathrm{CP}$ with teratoid features; $\mathrm{UI}=\mathrm{urinary}$ incontinence; $\mathrm{V}=$ visual deficit; $x$ antho $=$ xanthogranuloma; $3 \mathrm{~V}=$ third ventricle.

with suprasellar tumors without proven $\mathrm{CP}$ histology but whose clinicoradiological, surgical, and/or pathological features made it possible to conclude with near certainty the CP diagnosis. Classification of the cases in each subcohort is indicated in the far-right column in Table 1. The inclusion of this nonverified subcohort is required to provide a complete and coherent analysis of Cushing's series because a significant number of patients were treated with palliative surgical methods during the initial period of his career (1902-1917), making it impossible to pathologically confirm the diagnosis. Nevertheless, these cases were instrumental to Cushing in formulating the diagnostic criteria and surgical strategies that would be employed in subsequent periods. Therefore, with the aim of ensuring 
the reliability of our study, only the subcohort of verified $\mathrm{CPs}\left(\mathrm{CP}_{92}\right)$ is used for all analyses involving pathological variables, such as tumor topography.

\section{Variables Analyzed}

Table 2 shows the descriptive statistics for the epidemiological, clinical, diagnostic, and pathological variables analyzed in $\mathrm{CP}_{92}$ and the way in which these variables were categorized. The clinical symptoms in each patient were grouped into the following major syndromes: ${ }^{7}$ 1) high intracranial pressure, headache accompanied by vomiting, papilledema, and/or hydrocephalus; 2) chiasmal syndrome, loss of visual acuity or any visual field defect; 3) pituitary syndrome, symptoms of hypopituitarism, including isolated sexual disturbances, hair loss, atrophic skin alterations, and asthenia of unknown origin; 4) infundibulo-tuberal (IT) syndrome, Fröhlich's syndrome (sexual infantilism and obesity), changes in eating behavior, diabetes insipidus (DI), or sleep disturbances, in particular abnormal diurnal somnolence; and 5) hypothalamic syndrome, psychiatric, behavioral, or emotional alterations, including Korsakofflike memory impairment, abnormal changes in body temperature, gait disturbances, extrapyramidal symptoms, or sphincter incontinence.

\section{Topographical Tumor Classification}

Cushing's thorough descriptions of the anatomical CP-brain relationships, assessed both during the surgical procedure and in postmortem studies, provided precise written and/or pictorial information about the topography of these tumors in most patients (Fig. 2). We classified CP topography according to two major criteria: 1) anatomical compartments occupied by the lesion (sellar, suprasellar, and/or third ventricle [3V]); and 2) type of anatomical deformation of the 3V floor (3VF; integrity, displacement, or encroachment). Our interpretation of the anatomical CPbrain relationships provided by Cushing's surgical annotations and/or illustrative sketches was made in a consensual way by the two main authors (J.M.P. and R.P.). After incorporating this information into our topographical scheme, which has proven highly useful for predicting the severity of hypothalamic damage caused by tumor, ${ }^{28,29,32,33}$ we classified the CP topography in Cushing's series into five categories: 1) sellar/suprasellar CPs, which exclusively occupy the sellar and/or suprasellar compartments; 2) suprasellarpseudointraventricular CPs, which occupy the suprasellar cistern and cause upward displacement of the 3VF; 3) secondary intraventricular CPs, which extend from the sellar and/or suprasellar compartments into the $3 \mathrm{~V}$ after breaking through the 3VF; 4) IT or not strictly intraventricular CPs, tumors that develop in the $3 \mathrm{VF}$ and replace the infundibulum and tuber cinereum as they grow; and 5) strictly intraventricular CPs, which grow within the $3 \mathrm{~V}$, above an intact $3 \mathrm{VF}$. To ensure the accuracy and reliability of this scheme, the topographic analyses were only performed for $\mathrm{CP}_{92}$.

\section{Statistical Analysis}

Statistical analyses were performed using SPSS software (IBM SPSS Statistics for Windows, version 24.0, IBM Corp.). Frequencies and descriptive statistics on the
TABLE 2. Categorization of epidemiological, clinical, and pathological variables analyzed in Cushing's $\mathrm{CP}_{92}$ series

\begin{tabular}{|c|c|c|}
\hline Variable & No. of Cases (\%) & $\begin{array}{l}\text { No. of Cases } \\
\text { w/ Valid Data }\end{array}$ \\
\hline$M / F \operatorname{sex}$ & $45(48.9 \%) / 47(51.1 \%)$ & 92 \\
\hline $\begin{array}{l}\text { Age } \\
\quad \leq 19 \text { yrs } \\
20-49 \text { yrs } \\
\geq 50 \text { yrs }\end{array}$ & $\begin{array}{l}38(41.3 \%) \\
43(46.7 \%) \\
11(12 \%)\end{array}$ & 92 \\
\hline $\begin{array}{l}\text { Time course up to diagnosis } \\
\leq 6 \text { mos } \\
6 \text { mos }-1 \text { yr } \\
1-3 \text { yrs } \\
3-5 \text { yrs } \\
>5 \text { yrs }\end{array}$ & $\begin{array}{l}11(12.2 \%) \\
10(11.1 \%) \\
26(28.9 \%) \\
16(17.8 \%) \\
27(30 \%)\end{array}$ & 90 \\
\hline $\begin{array}{l}\text { Prevalence of major clinical } \\
\text { syndromes } \\
\text { HICP } \\
\text { Pituitary } \\
\text { Chiasmal } \\
\text { IT } \\
\text { Hypothalamic }\end{array}$ & $\begin{array}{l}61(66.3 \%) \\
62(67.4 \%) \\
74(80.4 \%) \\
38(41.3 \%) \\
24(26.1 \%)\end{array}$ & 92 \\
\hline \multicolumn{3}{|l|}{ Clinical variable } \\
\hline Headache & $86(93.5 \%)$ & 92 \\
\hline Visual deficits & $89(96.7 \%)$ & 92 \\
\hline Endocrine deficits & $70(76.1 \%)$ & 92 \\
\hline Fröhlich syndrome & $32(34.8 \%)$ & 92 \\
\hline Obesity & $37(40.2 \%)$ & 92 \\
\hline Cachexia & $8(8.7 \%)$ & 92 \\
\hline $\mathrm{DI}$ & $34(37.0 \%)$ & 92 \\
\hline Hypothalamic disturbances & $35(38.0 \%)$ & 92 \\
\hline Somnolence & $43(46.7 \%)$ & 92 \\
\hline Memory defect & $22(24.4 \%)$ & 90 \\
\hline Psychiatric disturbances & $43(46.7 \%)$ & 92 \\
\hline Gait disturbance & $16(17.4 \%)$ & 92 \\
\hline Neurological deficits & $20(21.7 \%)$ & 92 \\
\hline $\begin{array}{l}\text { Consciousness impairment } \\
\text { \&/or seizures }\end{array}$ & $19(20.7 \%)$ & 92 \\
\hline \multicolumn{3}{|l|}{ Diagnostic variables } \\
\hline $\begin{array}{l}\text { Diagnostic methodology } \\
\text { Clin \& ophthalmic } \\
\text { Clin, ophthalmic, \& Rx } \\
\text { Clin \& AirV }\end{array}$ & $\begin{array}{c}5(5.4 \%) \\
73(79.3 \%) \\
14(15.2 \%)\end{array}$ & 92 \\
\hline $\begin{array}{l}\text { Rx sign } \\
\text { Normal sella } \\
\text { Eroded sella outline } \\
\text { Enlarged \& eroded sella } \\
\text { Hyperostosis } \\
\text { S/SS calcifications }\end{array}$ & $\begin{array}{c}12(13.0 \%) \\
7(7.6 \%) \\
12(13.0 \%) \\
2(2.2 \%) \\
59(64.1 \%)\end{array}$ & 92 \\
\hline \multicolumn{3}{|l|}{ Pathological variables } \\
\hline $\begin{array}{l}\text { Tumor topography } \\
\text { S/SS } \\
\text { Pseudo 3V } \\
\text { S/SS-3V } \\
\text { IT } \\
\text { Strict 3V }\end{array}$ & $\begin{array}{c}16(17.4 \%) \\
20(21.7 \%) \\
30(32.6 \%) \\
21(22.8 \%) \\
5(5.4 \%)\end{array}$ & 92 \\
\hline
\end{tabular}


» CONTINUED FROM PAGE 954

TABLE 2. Categorization of epidemiological, clinical, and pathological variables analyzed in Cushing's $\mathrm{CP}_{92}$ series

\begin{tabular}{|c|c|c|}
\hline Variable & No. of Cases (\%) & $\begin{array}{l}\text { No. of Cases } \\
\text { w/ Valid Data }\end{array}$ \\
\hline \multicolumn{3}{|l|}{$\begin{array}{l}\text { Pathological variables (con- } \\
\text { tinued) }\end{array}$} \\
\hline Hydrocephalus & $58(63.0 \%)$ & 92 \\
\hline Tumor size & & 92 \\
\hline$<2 \mathrm{~cm}$ & $1(1.1 \%)$ & \\
\hline $2-3.9 \mathrm{~cm}$ & $47(51.1 \%)$ & \\
\hline $4-6 \mathrm{~cm}$ & $31(33.7 \%)$ & \\
\hline$>6 \mathrm{~cm}$ & $13(14.1 \%)$ & \\
\hline Tumor shape & & 89 \\
\hline Rounded & $21(23.6 \%)$ & \\
\hline Elliptical & $20(22.5 \%)$ & \\
\hline Multilobulated & $36(40.4 \%)$ & \\
\hline Pear-like & $12(13.0 \%)$ & \\
\hline Tumor consistency & & 92 \\
\hline Pure solid & $5(5.4 \%)$ & \\
\hline Pure cystic & $36(39.1 \%)$ & \\
\hline Mixed solid-cystic & $37(40.2 \%)$ & \\
\hline Cystic-basal solid & $14(15.2 \%)$ & \\
\hline Histological type & & 92 \\
\hline Not specified & $11(12.0 \%)$ & \\
\hline Adamantinomatous & $67(72.8 \%)$ & \\
\hline Squamous-papillary & $10(10.9 \%)$ & \\
\hline Mixed & $4(4.3 \%)$ & \\
\hline Degree of adherence* & & 82 \\
\hline Loose/dissectable & $8(9.8 \%)$ & \\
\hline Tight to sella/gland & $16(19.5 \%)$ & \\
\hline Tight to stalk/chiasm & $7(8.5 \%)$ & \\
\hline Tight to outer 3VF & $17(20.7 \%)$ & \\
\hline Ring-like to $3 \mathrm{VF} / 3 \mathrm{~V}$ & $18(22.0 \%)$ & \\
\hline Replacement of 3VF & $7(8.5 \%)$ & \\
\hline Tight to all structures & $9(11.0 \%)$ & \\
\hline Calcifications & $65(71.4 \%)$ & 91 \\
\hline Peritumoral gliosis & $54(71.1 \%)$ & 76 \\
\hline
\end{tabular}

* An accurate definition of CP adherence categories is provided in Prieto et al., 2016.

variables under study were obtained. Bilateral correlations between pairs of categorical variables were tested using the asymptotic chi-square test or the Monte Carlo exact test. Significance was assumed at a probability value less than 0.05 .

\section{Results}

\section{Cushing's CP Series: Demographic and Clinicopathological Features}

Table 1 presents a summary of the epidemiological, clinical, and pathological features of the 124 patients that form $\mathrm{CP}_{124}$. A rather balanced distribution between the sexes was observed (male/female ratio: 0.95 ), with $\mathrm{CP}$ incidence peaks in young patients 10-19 years old (29\%) and the other in middle-aged patients 30-49 years old
(25\%; Fig. 3A). The distribution of CP patients that Cushing treated in every 5 -year period throughout his career is shown in Fig. 3C. The incidence rates for the individual symptoms analyzed in $\mathrm{CP}_{124}$ are shown in Fig. 4A.

Within $\mathrm{CP}_{92}$, the three most common clinical syndromes observed at the time of diagnosis, isolated or in combination, were loss of vision caused by chiasm compression (80\%), hormonal deficits from pituitary insufficiency (67\%), and headache and/or vomiting due to high intracranial pressure (66\%; Table 2). Patients exhibited drowsiness in $47 \%$ of the cases, whereas a frankly comatose status occurred in $12 \%$. Clinical and/or radiological signs of obstructive hydrocephalus were identified in $63 \%$ of patients. Preoperative signs of DI were reported in $37 \%$. Interestingly, nearly half of the patients (47\%) manifested life-hampering neuropsychological or psychiatric disturbances, among them a severe cognitive deterioration associated with impaired short-term memory and confabulation similar to Korsakoff psychosis in $24 \%$.

The presumed preoperative diagnosis of $\mathrm{CP}$ was supported by findings of the funduscopic exam and skull radiography in $95 \%$ of patients. Within the $\mathrm{CP}_{92}$, abnormal funduscopy results were described in $93.5 \%$, with signs of retinal atrophy in 58\%, papilledema in $16 \%$, and papilledema superimposed on atrophy in $16 \%$. Skull radiographs displayed hyperdense shadows compatible with suprasellar calcifications in $64 \%$ of patients, an enlarged sella turcica in $13 \%$, and a normal-sized sella, with or without erosion of its outline, in $23 \%$. Additional air ventriculography exams were performed in only $15.2 \%$ of patients (Table 2).

Distribution of tumor topographies in the $\mathrm{CP}_{124}$ cohort is shown in Fig. 3B, and those corresponding to $\mathrm{CP}_{92}$ are described in Table 2 . The majority of tumors $(60.8 \%)$ in $\mathrm{CP}_{92}$ occupied the $3 \mathrm{~V}$ cavity at the time of surgery, either because they had developed primarily within the $3 \mathrm{VF}$ $(28 \%)$ or because they had broken through the $3 \mathrm{VF}$ from an original extraventricular position (33\%). The rest of the lesions had developed originally from the pituitary glandstalk complex, beneath the 3VF (39\%). Almost half of the tumors were large (over $4 \mathrm{~cm}$ ), and the predominant consistency was mixed solid-cystic (40\%). A multilobulated-shaped tumor was the most common morphology observed $(40.4 \%)$. The adamantinomatous histological variant was confirmed in $73 \%$ of the $\mathrm{CP}_{92}$ subcohort.

Strong tumor adherences to the $3 \mathrm{VF}$ or $3 \mathrm{~V}$ walls were observed during the surgical procedure in $62 \%$ of cases (Table 2). In the majority of strongly attached CPs, the tumor presented the most severe morphological pattern of adherence to the hypothalamus (ring-like attachment to the $3 \mathrm{VF}$ or replacement of the $3 \mathrm{VF}$ in $66.6 \%$ ), the ones associated with the highest surgical risk. ${ }^{38}$ This is consistent with the $71 \%$ rate of peritumoral gliosis observed in the histological studies.

\section{Relationships Between Epidemiological and Clinicopathological Variables}

Table 3 shows the most relevant relationships found between pairs of epidemiological, clinical, and/or topographical/pathological CP features within Cushing's series. The highest rate of endocrine deficits was observed in the pediatric population $(\mathrm{p}<0.001)$, whereas IT and hypo- 


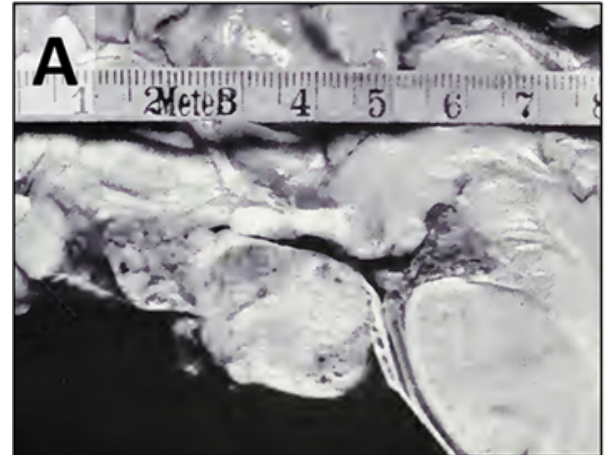

Sellar-Suprasellar

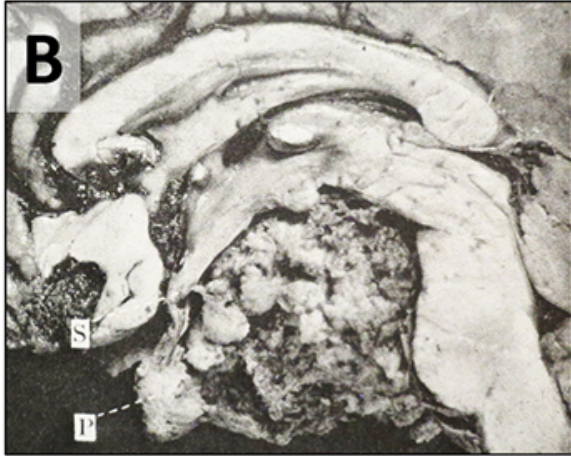

Pseudo-Intraventricular

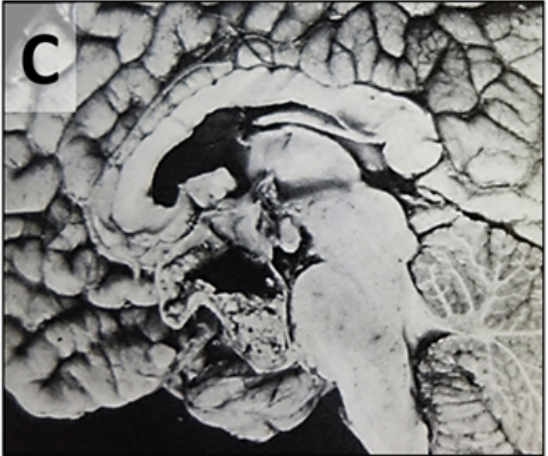

Suprasellar - Secondary Intraventricular

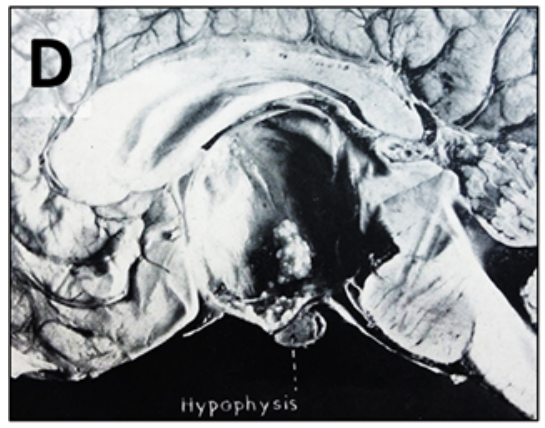

Infundibulo-tuberal

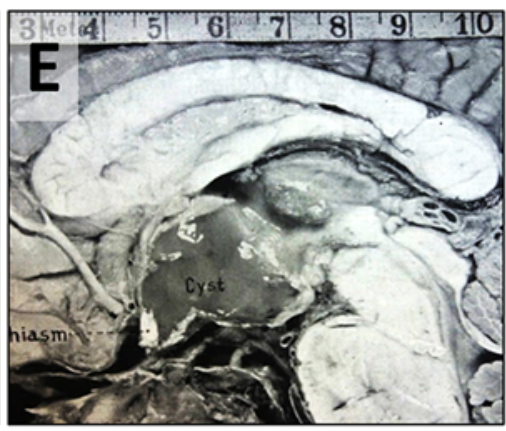

Strict 3V

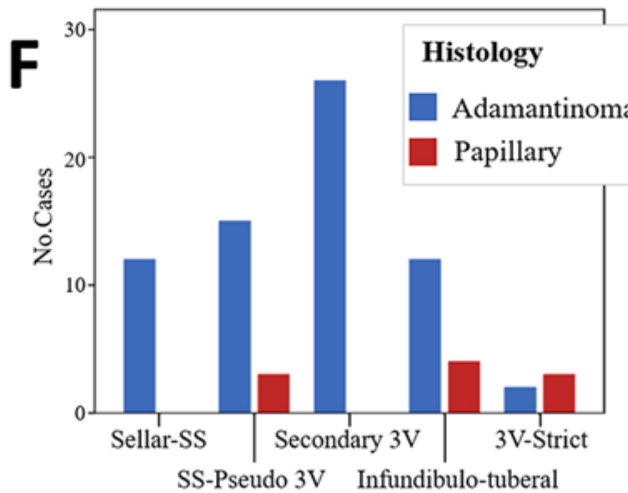

CP Topography

FIG. 2. Midsagittal tumor sections from autopsy specimens showing the five major tumor topographies evidenced in Cushing's $\mathrm{CP}_{92}$ series. A: Sellar/suprasellar: tumor occupies both the sellar and suprasellar compartments beneath an intact 3VF. B: Pseudo-intraventricular: this suprasellar tumor causes upward displacement of the 3VF, mimicking an intraventricular position. C: Sellar/ suprasellar-secondary intraventricular: a sellar and/or suprasellar lesion that eventually invades the $3 \mathrm{~V}$ after breaking through the 3VF (case 75). D: Infundibulo-tuberal: tumor develops within the nervous tissue of the 3VF (within the infundibulum and/or tuber cinereum) and predominantly expands within the $3 \mathrm{~V}$ (case 21). E: Strictly intraventricular: tumor primarily originates within the $3 \mathrm{~V}$, above an intact 3VF (case 6). Panels A, B, and E from Cushing: The Pituitary Body and Its Disorders, JB Lippincott, 1912. Public domain. Panel D from Walker: Arch Ophthalmol 45:407-437, 1916. Public domain. Panel C reproduced from Cushing's BTR. Public domain. Courtesy of the Manuscripts and Archives department of the Sterling Memorial Library, Yale University. F: Bar chart showing the relationship between the tumor topography and the histological variant in $\mathrm{CP}_{92}$. Note the lack of papillary cases among the sellar/suprasellar and secondary intraventricular topographies and the predominance of this histological variant in the group of strictly intraventricular CPs $(p<0.001) . S=$ sellar; $S S=$ suprasellar. Figure is available in color online only.

thalamic syndromes were mainly reported among adults older than 30 years of age $(p=0.01)$. Hydrocephalus occurred predominantly among children and young patients, a finding related to the development of giant $(\mathrm{p}=0.05)$ and multilobulated $(\mathrm{p}=0.04)$ tumors in these age groups (Fig. 4B). The squamous-papillary type was diagnosed almost exclusively among adults over 40 years old $(\mathrm{p}<0.001$; Fig. $4 C)$. In contrast, the highest rate of calcifications, typical of the adamantinomatous variant, occurred among patients under 20 years old $(\mathrm{p}<0.001)$.

\section{Clinical and Pathological Features Depending on CP Topography}

Craniopharyngioma topography was the variable showing the most robust relationships with the presence of certain groups of symptoms in the $\mathrm{CP}_{92}$ subcohort (Table $3)$. The highest rate of symptoms due to hypothalamic dysfunction, including Fröhlich syndrome, DI, abnormal somnolence, memory deficits, and psychiatric disturbances, was observed among CPs developing primarily within the 3VF (IT topography), as well as among those tumors invading the $3 \mathrm{~V}$ after breaking through the $3 \mathrm{VF}$ (sellar/ suprasellar-intraventricular topography; $\mathrm{p}<0.001$ ). Both topographies largely replaced the $3 \mathrm{VF}$ and presented the strongest and riskiest adherences to the hypothalamic remnants $(p<0.001)$, a fact linked to the highest rate of irreversible hypothalamic injury after Cushing's attempts to remove these types of lesions $(\mathrm{p}<0.001)$. The fundamental influence that CP topography had on patient outcome will be addressed in detail in Part 2 of this study.

\section{Discussion}

The "Interpeduncular Tumor": A Lesion Type That Marked Cushing's Career

A quirk of fate inescapably bound Harvey Cushing's 


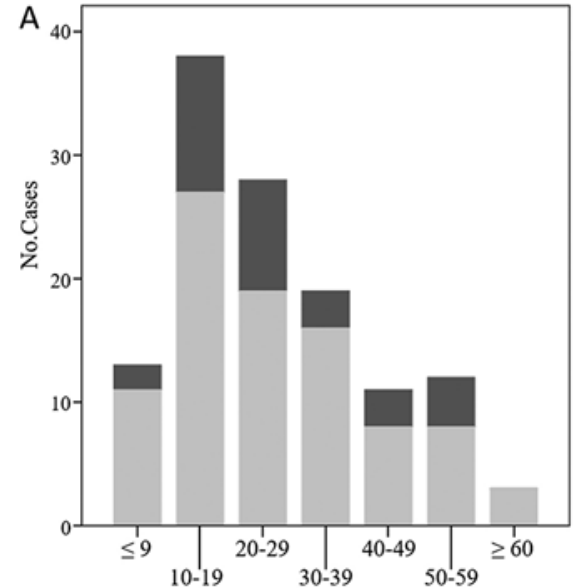

Age (years)

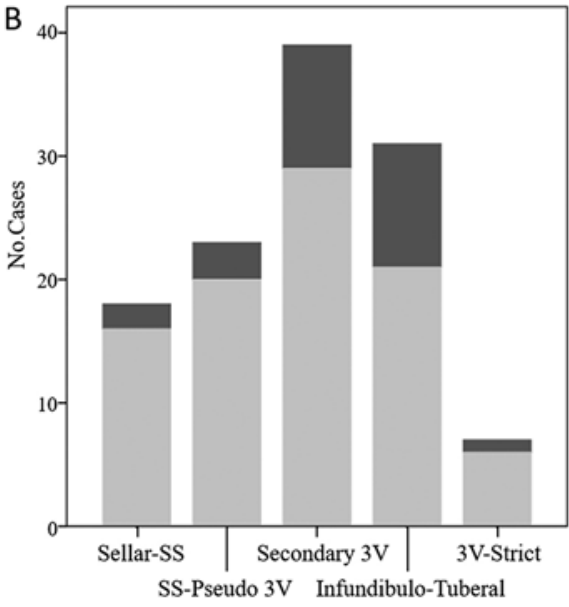

CP Topography

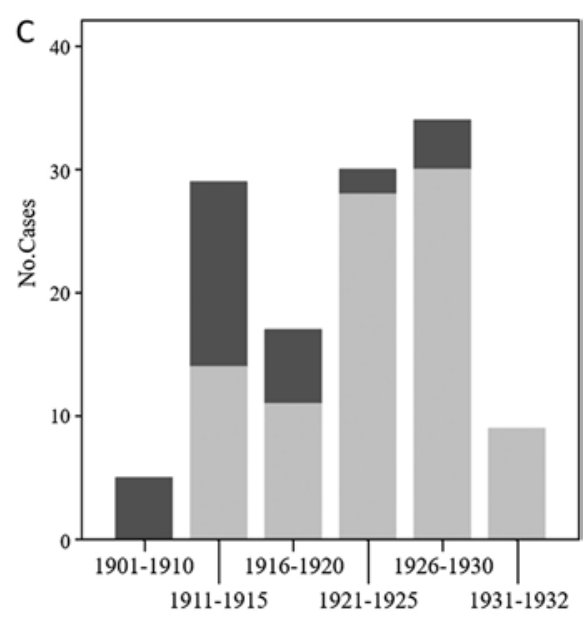

Cushing's surgical career

Series $\square$ Pathologically verified CPs $(n=92)$

Not verified CPs $(n=32)$

FIG. 3. Epidemiological and topographical characterization of Cushing's $\mathrm{CP}_{124}$ series. A: Histogram of age distribution. Half of the patients are between the ages of 10 and 29 years old. B: Bar graph showing the distribution of CP topographies. Sellar-SS = sellar/suprasellar (15\%); SS-Pseudo 3V = suprasellar pseudointraventricular (19\%); Secondary 3V = sellar/suprasellar-secondary intraventricular (33\%); Infundibulo-Tuberal = not strictly intraventricular (25\%); 3V-Strict = strictly intraventricular (5\%). C: Bar graph displaying the number of patients treated by Cushing for the time periods indicated. Note that the proportion of verified cases was higher from the 1920s onwards, coinciding with the refinement of diagnostic and histological methods. The low number of cases treated between 1916 and 1920 is due to Cushing's service during World War I.

career to CPs. Shortly upon his return from his year's sojourn in Europe, in December 1901, Cushing was confronted with his first "neurosurgical case" at Johns Hopkins, a kind of enigmatic intracranial lesion in a 16-yearold female named Mary D. ${ }^{14,16}$ This patient suffered from the same symptoms of sexual infantilism and obesity described by Alfred Fröhlich only a few months earlier, in a case that gave rise to the definition of Fröhlich's syndrome. ${ }^{22}$ Despite Cushing's efforts to alleviate his patient's severe intracranial pressure by performing three consecutive decompressive craniectomies, she eventually died, and a large, calcified cyst embedded in the infundibulum was found during her necropsy. Cushing drew an intricate sketch of the tumor, considered a teratoma at that time (Fig. 1B). Some years later, he acknowledged that the true pathological nature of this "interpeduncular tumor" was that of a CP. ${ }^{12,23,31}$ Eventually, the tumor specimen was lost, resulting in Cushing's determination to maintain all files and pathological specimens from his surgical patients, which served as the origin of the BTR, one of Cushing's greatest legacies and the source of information allowing for, a century later, the scientific analysis of his CP series.

\section{Cushing's Insights Toward a Precise Characterization of CPs}

At the time that Cushing published his acclaimed monograph on pituitary tumors in 1912, he had already treated eight interpeduncular tumors with discouraging results (cases 1-8; Table 1). ${ }^{14,18}$ Unlike pituitary gland "strumas" (i.e., macroadenomas), these interpeduncular lesions posed a formidable diagnostic and therapeu- tic challenge owing to their suprasellar location above a usually normal-sized sella turcica. ${ }^{30,34}$ Toward the end of 1911, Cushing found two epithelial cysts within the 3V in the brain specimens of two adults who had died, each after a short-term illness marked by severe psychiatric disturbances. ${ }^{14}$ Puzzled by the location and histology of these tumors, he named them "papillary infundibular cysts" in his pituitary monograph without acknowledging that they actually corresponded to the squamous-papillary variant of hypophyseal duct tumors, the entity categorized by the Austrian pathologist Jakob Erdheim (1874-1937) in 1904.21

Before Erdheim's seminal work defining the new category of hypophyseal duct tumors (CPs), a great number of rather confusing terms had been employed to describe the suprasellar tumors with the pathological features typical of CPs. ${ }^{6,35}$ Many of these lesions were included under the common category of "pituitary cysts," whereas most of them actually originated above an intact pituitary gland. Aware of this fact, Cushing favored the anatomical over the histological viewpoint by initially adopting the term "suprasellar cyst" to refer to the type of interpeduncular tumor he had first identified in 1901. Between 1911 and 1921, he used the terms "suprasellar" and "interpeduncular" interchangeably to refer to the group of congenital tumors presumably derived from cell inclusion (anlage) of either Rathke's pouch or its ascending buccal-cranial pathway, the hypophyseal duct in early embryonic life. ${ }^{14,18}$ It appears that Cushing purposely avoided the term "hypophyseal duct tumor" introduced by Erdheim, despite knowing of and mentioning Erdheim's paper in his 1912 magnum opus on the pituitary gland.${ }^{14}$ Curiously, Percival Bailey (1892-1973), a neurosurgical trainee of Cushing, 


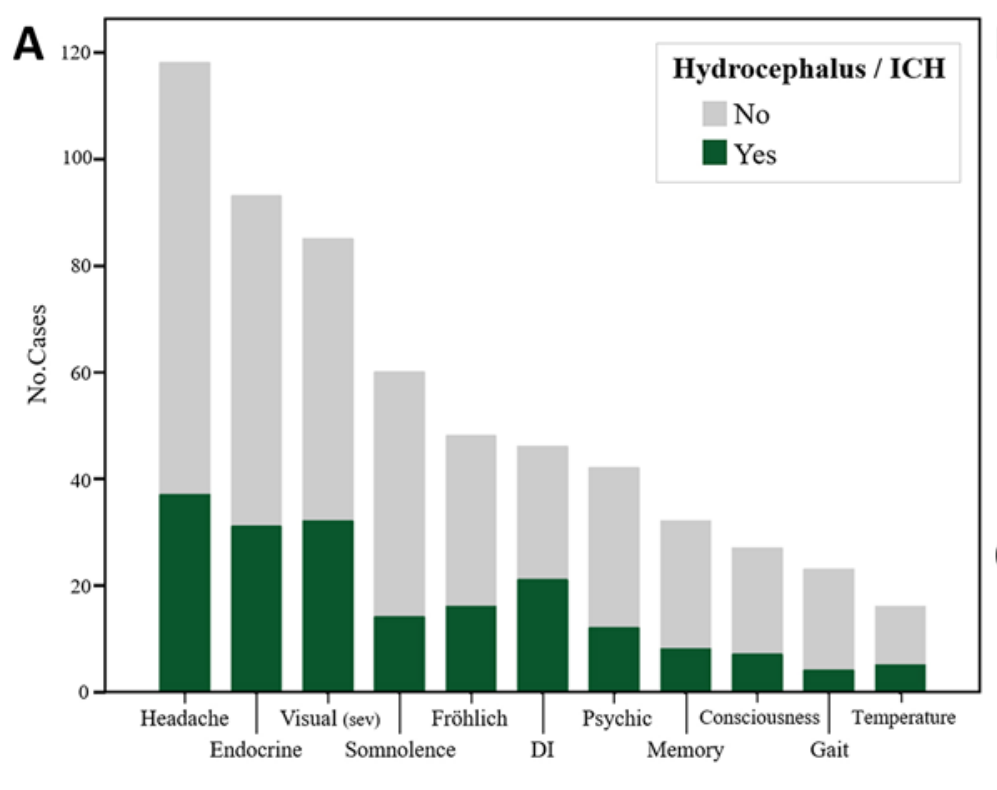

Symptoms
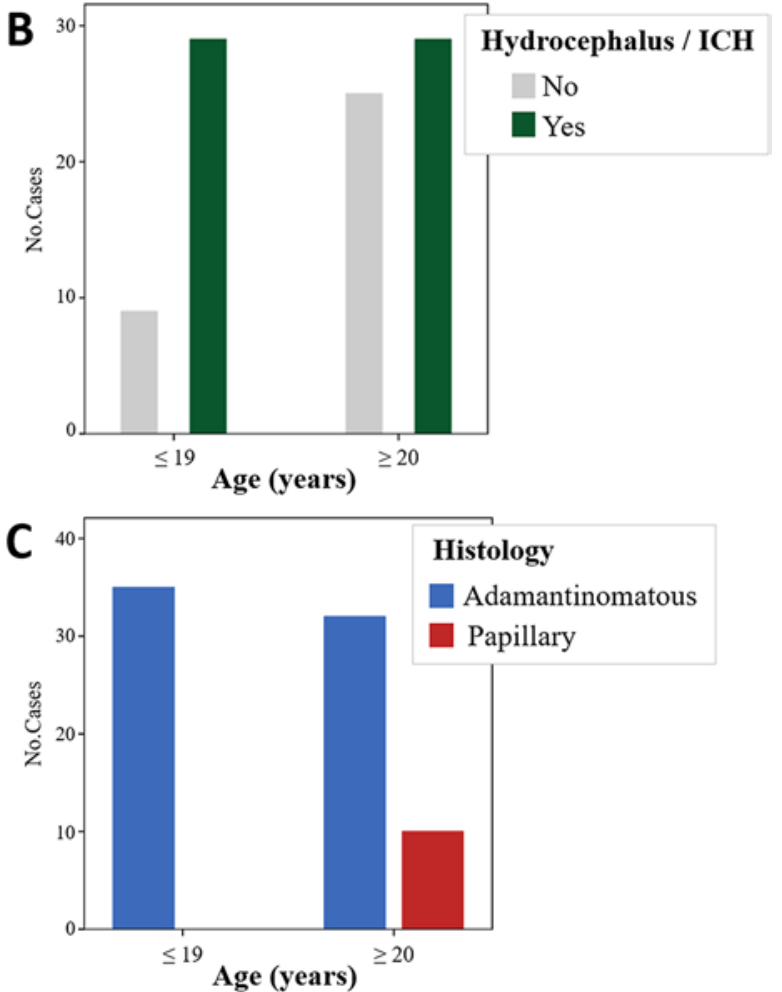

FIG. 4. Clinical and histological characterization of Cushing's CP series. A: Bar graph displaying the distribution of the main clinical symptoms in $\mathrm{CP}_{124}$. Dark green area within each bar represents the cases within each category presenting with hydrocephalus or symptoms of intracranial hypertension (ICH). Headache (95\%), endocrine deficits (76\%), and severe visual deficits (Visual [sev]; 68\%) were the most common symptoms in this cohort. B: Correlation between age and the presence of hydrocephalus or symptoms of $\mathrm{ICH}$ in $\mathrm{CP}_{92}$. Note that $76 \%$ of patients under 20 years of age suffered from ICH symptoms $(p<0.001)$. C: Relationship between age and histological variant in the $\mathrm{CP}_{92}$. Note that all papillary CPs were diagnosed in patients $\geq 20$ years of age $(p<$ 0.001). Figure is available in color online only.

continued to employ Erdheim's term in his pathological studies on Cushing's CP cases. ${ }^{1,3,37}$

Throughout most of the 1920s, Cushing preferentially employed the misnamed terms "pharyngeal pouch cyst" or "craniopharyngeal pouch cyst" despite the well-established concept that the adenohypophyseal primordium, or Rathke's pouch, originates from the embryo's primitive buccal cavity (stomodeal epithelium), not from the pharynx. ${ }^{27,39}$ Cushing himself picked the catchy term "craniopharyngioma" in 1929, adding the Greek suffix "-oma" (meaning "swelling" or "tumor") to the combination of "cranio" and "pharynx," and he employed it for the first time in the patient B.L.E. (case 110; Table 1 and Fig. 1C). $\mathrm{He}$ chose this designation to highlight the congenital nature of these extremely heterogeneous, epithelial tumors, presumably derived from the pituitary primordium. This concept was practically the same as the one proposed by Jakob Erdheim. ${ }^{36}$ Cushing incorporated this new term into his three major monographs addressing this type of tumor; thus, this moniker spread worldwide and ultimately prevailed over Erdheim's terminology. ${ }^{9,11,13}$

\section{Cushing's Clinical Assessment of CP Patients: An Open Window Into the Workings of the Brain}

Craniopharyngioma treatment posed enormous tech- nical challenges to Harvey Cushing, yet it also provided an endless supply of insight regarding a wide range of endocrinological and neuropsychological disturbances caused by these tumors. ${ }^{2,13,24}$ As a type of lesion primarily involving the infundibulum and adjacent hypothalamus, CPs definitely served to shed light on the separation of hypothalamic and hypophyseal functions. ${ }^{7,31,32}$ Alfred Fröhlich's 1901 identification of the adiposogenital syndrome enabled Cushing to preoperatively diagnose CPs, especially among children and young patients manifesting DI (Fig. 5A1 and A2). ${ }^{22}$ But rather than suspecting hypothalamic dysfunction, Cushing initially considered Fröhlich's syndrome to involve tumors blocking the passage of pituitary gland secretions into the $3 \mathrm{~V}$, secretions he thought were essential for adequate brain control of body homeostasis. ${ }^{31}$ Notably, it was Percival Bailey who proved incontrovertibly that DI and Fröhlich's syndrome were generated by lesions restricted to the hypothalamus. ${ }^{4}$ Bailey also provided the important concept that a specific set of symptoms observed in CP patients, such as hypersomnia and personality and/or behavioral changes, represents true hypothalamic alterations. ${ }^{2}$ In his 1930 lecture to the Royal College of Surgeons in London, Cushing acknowledged Bailey's important contributions and summed up the clinical and anatomical evidence provided by $\mathrm{CP}$ patients regarding the newly recognized "hypothalamic 
TABLE 3. Bivariate relationships between epidemiological, topographical, and pathological categories analyzed for Cushing's surgically treated CP series, 1901-1932

\begin{tabular}{|c|c|c|}
\hline Variable & $\mathrm{p}$ Value & Specific Relationship \\
\hline \multicolumn{3}{|l|}{ Related to age $\left(\mathrm{CP}_{124}\right)$} \\
\hline Hydrocephalus & $<0.001$ & Highest rate of hydrocephalus in patients younger than $30 \mathrm{yrs}$ \\
\hline Major syndrome & 0.01 & Highest rate of IT/hypothalamic syndrome in patients older than $30 \mathrm{yrs}$ \\
\hline Endocrine symptoms & $<0.001$ & Highest rate of endocrine deficits in group $10-19$ yrs old \\
\hline Memory disturbances & 0.029 & Highest rate among adult patients older than $20 \mathrm{yrs}$ \\
\hline CP size & 0.05 & Highest rate of large lesions $(4-10 \mathrm{~cm})$ in pediatric group (age $<20 \mathrm{yrs})$ \\
\hline CP shape & 0.04 & Highest rate of multilobulated lesions in pediatric group (age $<20 \mathrm{yrs}$ ) \\
\hline CP histology & $<0.001$ & Highest rate of squamous-papillary variant in group $40-69$ yrs old \\
\hline CP calcifications & $<0.001$ & Highest rate of calcifications in pediatric group (age $<20 \mathrm{yrs}$ ) \\
\hline \multicolumn{3}{|l|}{ Related to $\mathrm{CP}$ topography $\left(\mathrm{CP}_{92}\right)$} \\
\hline ITS & $<0.001$ & Highest rate in IT \& S/SS-3V CPS \\
\hline Hypothalamic syndrome & $<0.001$ & Highest rate in S/SS-3V \& 3V CPS \\
\hline Fröhlich syndrome & 0.029 & Highest rate in IT \& S/SS-3V CPS \\
\hline Psychiatric disturbances & 0.03 & Highest rate in IT, S/SS-3V, \& 3V CPs \\
\hline Somnolence & $<0.001$ & Highest rate in IT \& 3V CPs \\
\hline Memory disturbances & $<0.001$ & Highest rate in S/SS-3V \& IT CPS \\
\hline Consciousness disturbances & 0.002 & Present almost exclusively in S/SS-3V \& IT CPS \\
\hline DI & 0.06 & Highest rate among IT CPs \\
\hline Hydrocephalus & $<0.001$ & Highest rate of hydrocephalus w/ S/SS-3V CPs \\
\hline CP shape & $<0.001$ & Highest rate of rounded lesions among IT CPS \\
\hline CP histology & 0.007 & Highest rate of squamous-papillary type in IT and $3 \mathrm{~V}$ CPs \\
\hline CP adherence & $<0.001$ & Replacement of 3VF only in IT CPs \\
\hline \multicolumn{3}{|l|}{ Related to $\mathrm{CP}$ pathological features $\left(\mathrm{CP}_{92}\right)$} \\
\hline CP size \& tumor adherence & 0.01 & Highest rate of strong adherence to $3 \mathrm{~V}$ in $\mathrm{CPs} 4-6 \mathrm{~cm}$ \\
\hline CP consistency \& tumor adherence & 0.002 & $\begin{array}{l}\text { Highest rate of strong adherence to all brain structures in mixed solid-cystic } \\
\text { tumors; highest rate of } 3 V F \text { replacement in cystic-basal solid CPs }\end{array}$ \\
\hline CP histology \& adherence & 0.008 & $\begin{array}{l}\text { Highest rate of strong } \mathrm{CP} \text { adherences in adamantinomatous type; highest rate of } \\
\text { pedicle attachment in squamous-papillary type }\end{array}$ \\
\hline CP adherence \& postop hypothalamic injury & 0.003 & $\begin{array}{l}\text { No hypothalamic injury when CPs have loose adherence; hypothalamic injury in } \\
>85 \% \text { of CPs w/ } 3 \text { VF replacement }\end{array}$ \\
\hline
\end{tabular}

doctrine" for a central, hypothalamic control of body metabolism and water homeostasis..$^{13}$

Harvey Cushing was also the first author to take interest in the behavioral and psychological disturbances manifested by CP patients..$^{15}$ Clinical and pathological evidence provided by Fulton and Bailey in 1929 conclusively proved that these symptoms had a primarily hypothalamic origin. ${ }^{24}$ The analysis of Cushing's entire CP series shows that the rate of patients manifesting severe pre- or postoperative neuropsychological or psychiatric disturbances that hampered their quality of life to the point of forcing them to quit their jobs or school and/or affected their interpersonal relationships reached 45\% (57 patients). Among these disturbances, severe cognitive deterioration including a marked short-term memory deficit similar to Korsakoff psychosis (in $24 \%$ of the $\mathrm{CP}_{124}$ ), a status of emotional lability including rage attacks (in 16\%), and/or an apathetic attitude or permanent disinterest in their own lives (in 9\%) were the predominant alterations. Such disturbances were chiefly observed in patients with CPs developing primar- ily within the $3 \mathrm{VF}$ (in $33 \%$ of IT CPs) and in large tumors invading the $3 \mathrm{~V}$ (in $44 \%$ of $3 \mathrm{~V}$-invading $\mathrm{CPs}$ ).

\section{Cushing's Methodology and Rationale for CP Diagnosis}

Craniopharyngioma diagnosis during Cushing's time at the Peter Bent Brigham Hospital (1912-1932) involved a great deal of uncertainty. The only available methods for the preoperative diagnosis of pituitary tumors were systematic clinical and neurological explorations such as funduscopic exams and identification of sella turcica alterations on skull radiographs. ${ }^{10,16,30,34}$ Despite the rudimentary nature of these tools, Harvey Cushing decisively contributed to the refinement of the clinical and radiological clues allowing for differentiation between pituitary adenomas and CPs with reasonable accuracy.

Ophthalmoscopic retinal exams were fundamental for Cushing to successfully develop a neurosurgical program for pituitary tumors. He soon learned how to identify the presence of choked discs (papilledema), the objective sign of high intracranial pressure through which the presence 

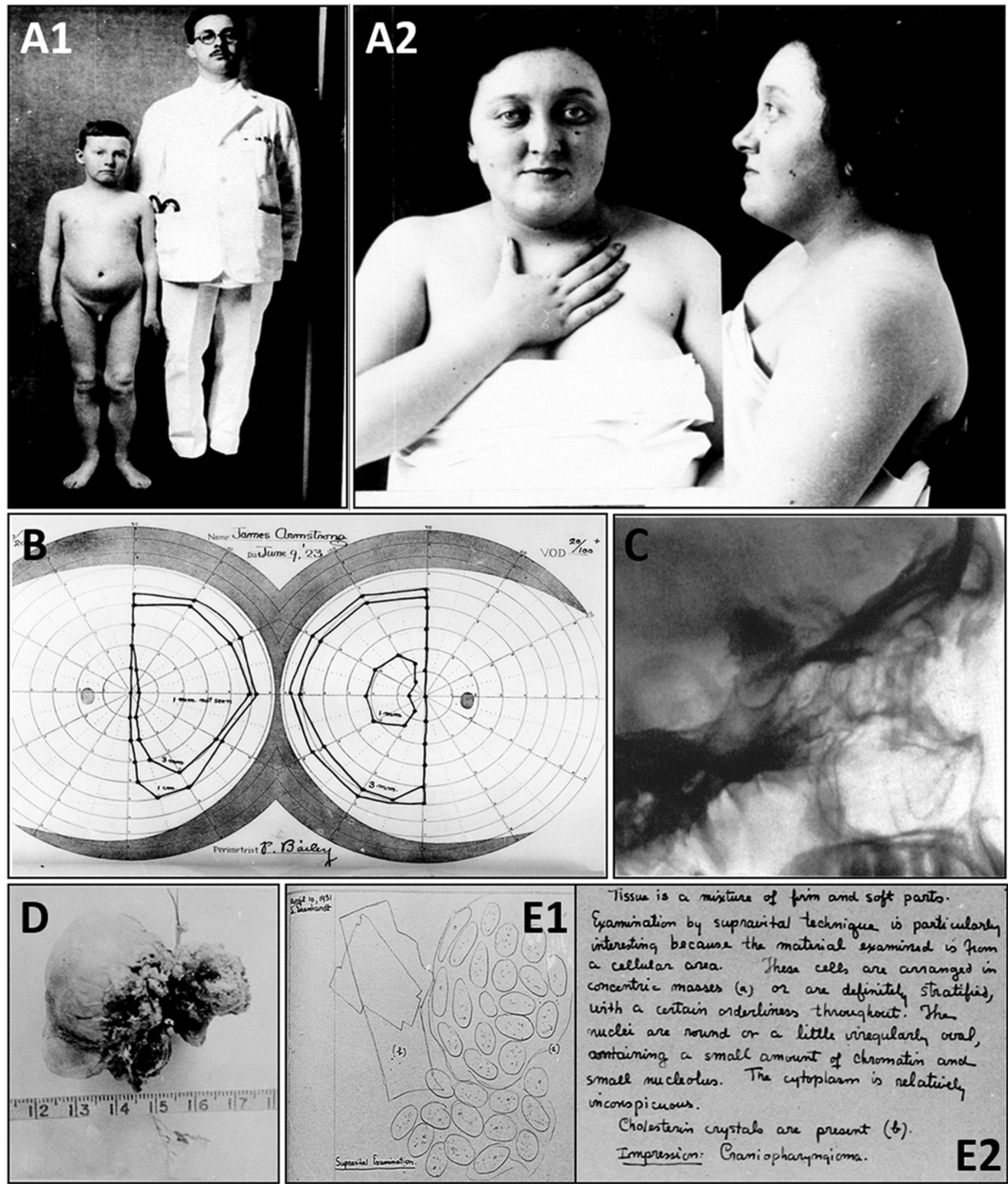

Tissur is a mixture of frim and soft parts.

Examination by supravital technique is particularly interesting because the material examinad is from a cellular area. These cells are arranged in concentric masses (a) or are definitel stratified, with a certain anderliness throughout. The nuclei are round on a litte virequlares oval, containing a small amount of chromation and small nucleolus. The cytoplasm is selatively incosspicuous.

Cholesterin crystab are present (b).

Impression: Ganiopharymaioma.

FIG. 5. Harvey Cushing's methodology for CP diagnosis. Fröhlich's syndrome phenotype: a 12-year-old boy with incipient feminine-type obesity and failed sexual development (J.A.D., case 64, A1) and a young woman, 19 years old, with amenorrhea and marked obesity (H.B., case 23, A2). Perimetry exam showing bitemporal hemianopia, a visual defect characteristic of the optic chiasm injury caused by CPs (J.C.A., case 9, B). Lateral skull radiograph of J.A.B. (case 92, C), obtained in October 1926, showing dense suprasellar shadows just above the sella turcica, the radiological correlate of tumor calcifications. Picture of tumor specimen removed from D.A. (case 17, D) in June 1913. FIG. 5. (continued) $\rightarrow$ 
FIG. 5. Note the multilobulated, solid-cystic structure of the lesion, whose hard capsule was tightly adhered to the vessels of the circle of Willis. This patient died shortly after tumor removal because of the severe intraoperative bleeding and permanent hypothalamic injury caused by en bloc resection of the mass. Sketch of the microscopic appearance of a typical CP, created by Louise Eisenhardt (1891-1967), Cushing's associate neuropathologist, on April 10, 1931 (S.G.W., case 117, E1). Examination of the solid tumor portion via the supravital technique showed epithelial cells arranged in concentric masses or whorls (a) and cholesterin crystals (b). Handwritten description (E2) of histological characteristics of tumor featured in E1, by Louise Eisenhardt. Reproduced from Cushing's BTR. Public domain. Courtesy of the Manuscripts and Archives department of the Sterling Memorial Library, Yale University.

of an intracranial tumor could be presumed. ${ }^{34,35}$ However, the observation of macular atrophy instead of papilledema was a finding typically reported for CP patients. ${ }^{40}$ This localizing sign, indicating direct optic chiasm compression by a suprasellar mass, in combination with symptoms of hypophyseal insufficiency, helped Cushing to differentiate suprasellar lesions from large intracranial tumors involving other locations.

Clifford B. Walker (1884-1943), an ophthalmologist collaborating with Cushing at Peter Bent Brigham, described the specific campimetric defects associated with optic chiasm distortions by pituitary tumors. ${ }^{40}$ Bitemporal hemianopia was identified as a rather specific visual deficit due to injury caused to the central portion of the chiasm by the tumor bulk (Fig. 5B). It was observed in 26 of 81 patients with large hypophyseal and parahypophyseal tumors. Four of these cases corresponded to CPs. After these studies, routine campimetric and funduscopic exams were performed for the majority of Cushing's CP patients, as reported in the BTR. Bitemporal and concentric campimetric deficits associated with optic atrophy in patients manifesting the triad of severe headache, loss of vision, and Fröhlich's syndrome formed the basis for the clinical suspicion of CP. ${ }^{34}$

In 1914, an important radiological study by Alfred Luger (1886-1938), the first full-time radiologist at the Peter Bent Brigham Hospital, identified the distinctive radiographic sign of nodular hyperdense shadows above or within the sella turcica in three CPs, the correlate of calcareous depositions within the tumor. ${ }^{25}$ Merrill Clary Sosman (1890-1959), a neuroradiologist who started collaborating with Cushing in 1922, significantly improved the detection of intracranial calcifications with skull radiographs and, in 1924, conclusively proved the value of this sign for accurate preoperative diagnosis of calcified CPs, which represented $71 \%$ of the cases in Cushing's series (Fig. 5C). ${ }^{26}$

Cushing's use of air ventriculography for CP diagnosis would be postponed until the final stage of his career. ${ }^{34}$ Cushing first tested this method in 1919 (case 45; Table 1), yet his personal animosity toward Walter Dandy (1886-1946), his former trainee at Johns Hopkins and the inventor of air ventriculography in 1918, undoubtedly influenced his dismissal of this valuable technique, which he only used occasionally before $1928 .{ }^{20,41}$ Afterward, Cushing acknowledged its utility in outlining $\mathrm{CP}$ boundaries as well as $3 \mathrm{~V}$ occupation by the tumor. Air ventriculography would prove essential for implementing the new intraventricular approaches employed by Cushing to gain direct access to $3 \mathrm{~V}$ CPs. In his last monograph on meningiomas, published in 1938, Cushing praised "Dr. Dandy's brilliant and useful discovery" (page 522) and admitted that he had restricted its use because of an overly conservative attitude and unfounded fear regarding the risks associated with air ventriculography. ${ }^{19}$

\section{Hypothalamus Involvement in Cushing's CP Series: The Major Determinant of Surgical Risk}

For a long period, Cushing overlooked the anatomical evidence that many CPs occupied a high position within the infundibulum and $3 \mathrm{~V}$, as confirmed in many necropsy studies in his own patients (Figs. 1B and 2A-E). Although he initially rejected Erdheim's claims that CPs primarily involved neural centers at the base of the $3 \mathrm{~V}$ (i.e., the hypothalamus), ${ }^{21}$ roughly $60 \%$ of the tumors in $\mathrm{CP}_{92}$ primarily expanded within the hypothalamus (IT and $3 \mathrm{~V} \mathrm{CPs}$ ) or had encroached upon this region from the sellar/suprasellar compartments (secondarily intraventricular CPs; Fig. 2C-E). Most of these patients manifested symptoms related to hypothalamic dysfunction. After a systematic examination of Cushing's CP autopsy specimens, Percival Bailey was unable to discriminate tumor boundaries from the adjacent hypothalamus; consequently, he considered these lesions as true "tumors of the hypothalamus."

The extensive, strong tumor adherences to the hypothalamus usually present in CPs involving the $3 \mathrm{~V}$ represent a critical factor, accounting for the high mortality associated with the surgical removal of these tumors in Cushing's series (Fig. 2D and Table 3). ${ }^{38}$ Early on, Cushing noticed the intractable hyperthermia and permanent lethargic condition that usually followed surgical attempts at $\mathrm{CP}$ removal when the tumor strongly adhered to the hypothalamic region. In $90 \%$ of the deaths occurring in the first 8 weeks after surgery ( 25 of 28 patients), mortality was directly related to hypothalamic damage. This injury resulted from the removal of portions of the hypothalamus strongly adhered to the tumor capsule (12 patients) or was attributable to a delayed hypothalamic failure some days or weeks after the procedure, caused by ischemic or hemorrhagic diencephalic injury (Fig. 2D). Postoperative hypothalamic dysfunction could not be offset with corticosteroids or hormone replacement therapy at that time. Accordingly, Cushing's goal was to find new surgical methods to provide optimal visualization and safe dissection of CPs tightly attached to the hypothalamus. ${ }^{31,37}$ These methods will be properly addressed in Part 2 of this work.

\section{Conclusions}

Analysis of Cushing's CP series is meaningful for neurosurgeons today as it shows the nature of the main problem posed by these complex lesions: the high risk of hypothalamic injury associated with their removal. The clinical insights, pathophysiological concepts, and diag- 
nostic methodology implemented by Harvey Cushing in $\mathrm{CP}$ treatment contributed much to establishing the foundation for modern neurosurgical understanding of this tumor type. Cushing's legacy to modern $\mathrm{CP}$ neurosurgery can be summed up in four key points: 1) the categorization of clinical symptoms specifically derived from either hypothalamic dysfunction or hypophysial insufficiency; 2) the accurate description of anatomical CP-brain relationships; 3 ) the development of a rational protocol for $\mathrm{CP}$ topography diagnosis based on neuroophthalmological and radiological data; and 4) the exploration of all possible surgical avenues to these deep-seated tumors, including the set of intraventricular techniques necessary for the safe removal of CPs involving the hypothalamus.

\section{Acknowledgments}

We wish to especially thank Bill Landis and all the staff of the Manuscripts and Archives department of the Sterling Memorial Library, Yale University (New Haven, CT), for their kind assistance in accessing the Cushing Brain Tumor Registry. We are also grateful to Terry Dagradi, coordinator and curator of the Cushing Center at the Cushing/Whitney Medical Library, Yale University (New Haven, CT), for her kind guidance and assistance during our visit to the Cushing Center. We are indebted to Crystal Smith and Liliya Gusakova, reference librarians of the National Library of Medicine, National Institutes of Health (Bethesda, MD), and Jack Eckert and the staff of the Francis Countway Medical Library at the Harvard Medical School (Boston, MA), for their generous assistance during the process of searching for and retrieving some of the original research material used in this study. Finally, we wish to express our gratitude to George Hamilton (who was born at Brigham and Women's Hospital, formerly Peter Bent Brigham Hospital) for his critical review of the language and style of the manuscript.

\section{References}

1. Bailey P: Concerning the cerebellar symptoms produced by suprasellar tumours. Arch Neurol Psychiatry 11:137-149, 1924

2. Bailey P: Craniopharyngiomas. Syndrome of the hypothalamus, in: Intracranial Tumors. Springfield, IL: Charles H. Thomas, 1933, pp 113-137

3. Bailey P: Notes concerning keratin and keratohyalin in tumors of the hypophysial duct. Ann Surg 74:501-505, 1921

4. Bailey P, Bremer F: Experimental diabetes insipidus. Arch Intern Med 28:773-803, 1921

5. Bailey P, Cushing H: A Classification of the Tumors of the Glioma Group on a Histogenetic Basis with a Correlated Study of Prognosis. Philadelphia: JB Lippincott, 1926

6. Boyce R, Beadles CF: A further contribution to the study of the pathology of the hypophysis cerebri. J Pathol Bacteriol 1:359-383, 1893

7. Castro-Dufourny I, Carrasco R, Prieto R, Barrios L, Pascual JM: The infundibulo-tuberal syndrome caused by craniopharyngiomas: clinicopathological evidence from an historical French cohort (1705-1973). Pituitary 18:642-657, 2015

8. Cohen-Gadol AA, Spencer DD (eds): The Legacy of Harvey Cushing. Profiles of Patient Care. New York: Thieme, 2007, pp 1-93

9. Cushing H: The chiasmal syndrome of primary optic atrophy and bitemporal field defects in adults with normal sella turcica. Arch Ophtalmol (Paris) 3:505-551, 1930

10. Cushing H: The hypophysis cerebri: clinical aspects of hyperpituitarism and of hypopituitarism. JAMA 53:249-255, 1909
11. Cushing H: Intracranial Tumors. Notes Upon a Series of Two Thousand Verified Cases With Surgical Mortality Percentages Pertaining Thereto. Springfield, IL: Charles C. Thomas, Springfield, IL 1932, pp 93-98

12. Cushing H: Les syndromes hypophysaires au point de vue chirurgical. Rev Neurol (Paris) 29:779-808, 1922

13. Cushing H: Papers Relating to the Pituitary Body, Hypothalamus and Parasympathetic Nervous System. Springfield, IL: Charles C. Thomas 1932, pp 43-56

14. Cushing H: The Pituitary Body and Its Disorders. Philadelphia: JB Lippincott, 1912

15. Cushing H: Psychic disturbances associated with disorders of the ductless glands. Am J Insanity 69:965-990, 1913

16. Cushing H: Sexual infantilism with optic atrophy in cases of tumor affecting the hypophysis cerebri. J Nerv Ment Dis 33:704-716, 1906

17. Cushing H: Tumors of the Nervus Acusticus and the Syndrome of the Cerebellopontine Angle. Philadelphia: WB Saunders Company, 1917

18. Cushing H: The Weir Mitchell Lecture. Surgical experiences with pituitary disorders. JAMA 63:1515-1525, 1914

19. Cushing H, Eisenhardt L: Meningiomas. Their Classification, Regional Behaviour, Life History and Surgical End Results. Springfield, IL: Charles C. Thomas, 1938

20. Dandy WE: Ventriculography following the injection of air into the cerebral ventricles. Ann Surg 68:5-11, 1918

21. Erdheim J: Über Hypophysengangsgeschwulste und Hirmcholesteatome. Sitzungsb Kais Akad Wissen Math Naturw Klin 113:537-726, 1904

22. Fröhlich A: Ein fall von tumor der hypophysis cerebri ohne akromegalie. Wien Klin Rundschau 15:883-886, 906-908, 1901

23. Fulton JF: Harvey Cushing. A Biography. Springfield, IL: Charles C. Thomas, 1946

24. Fulton JF, Bailey P: Tumors in the region of the third ventricle: their diagnosis and relation to pathological sleep. J Nerv Mental Dis 69:1-25, 145-164, 261-277, 1929

25. Luger A: Zur Kenntnis der im Röntgenbild sichtbaren Hirntumoren mit besonderer Berücksichtigung der Hypophysengangsgeschwülste. Fortschr Röntgenstr21: 605-614, 1914

26. McKenzie KG, Sosman MC: The roentgenological diagnosis of craniopharyngeal pouch tumors. AJR Am J Roentgenol 11:171-176, 1924

27. McLean JA: Die Craniopharyngealtaschentumoren (Embryologie, Histologie, Diagnose und Therapie). Zeitschr Ges Neurol Psych 126:639-682, 1930

28. Pascual JM, Carrasco R, Prieto R, Gonzalez-Llanos F, Alvarez F, Roda JM: Craniopharyngioma classification. J Neurosurg 109:1180-1183, 2008

29. Pascual JM, González-Llanos F, Barrios L, Roda JM: Intraventricular craniopharyngiomas: topographical classification and surgical approach selection based on an extensive overview. Acta Neurochir (Wien) 146:785-802, 2004

30. Pascual JM, Mongardi L, Prieto R, Castro-Dufourny I, Rosdolsky M, Strauss S, et al: Giovanni Verga (1879-1923), author of a pioneering treatise on pituitary surgery: the foundations of this new field in Europe in the early 1900s. Neurosurg Rev 40:559-575, 2017

31. Pascual JM, Prieto R: Harvey Cushing and pituitary Case Number 3 (Mary D.): the origin of this most baffling problem in neurosurgery. Neurosurg Focus 41(1):E6, 2016

32. Pascual JM, Prieto R, Carrasco R: Infundibulo-tuberal or not strictly intraventricular craniopharyngioma: evidence for a major topographical category. Acta Neurochir (Wien) 153:2403-2426, 2011

33. Pascual JM, Prieto R, Carrasco R, Barrios L: Displacement of mammillary bodies by craniopharyngiomas involving the third ventricle: surgical-MRI correlation and use in topographical diagnosis. J Neurosurg 119:381-405, 2013 
34. Pascual JM, Prieto R, Castro-Dufourny I, Carrasco R, Strauss S, Barrios L: Development of intracranial approaches for craniopharyngiomas: an analysis of the first 160 historical procedures. Neurosurg Focus 36(4):E13, 2014

35. Pascual JM, Prieto R, Mazzarello P: Sir Victor Horsley: pioneer craniopharyngioma surgeon. J Neurosurg 123:39-51, 2015

36. Pascual JM, Rosdolsky M, Prieto R, Strau $\beta$ S, Winter E, Ulrich W: Jakob Erdheim (1874-1937): father of hypophyseal-duct tumors (craniopharyngiomas). Virchows Arch 467:459-469, 2015

37. Prieto R, Pascual JM: Norman M. Dott, master of hypothalamic craniopharyngioma surgery: the decisive mentoring of Harvey Cushing and Percival Bailey at Peter Bent Brigham Hospital. J Neurosurg 127:927-940, 2017

38. Prieto R, Pascual JM, Rosdolsky M, Castro-Dufourny I, Carrasco R, Strauss S, et al: Craniopharyngioma adherence: a comprehensive topographical categorization and outcomerelated risk stratification model based on the methodical examination of 500 tumors. Neurosurg Focus 41(6):E13, 2016

39. Solov'ev GS, Bogdanov AV, Panteleev SM, Yanin VL: Embryonic morphogenesis of the human pituitary. Neurosci Behav Physiol 38:829-833, 2008

40. Walker CB, Cushing H: Studies of optic-nerve atrophy in association with chiasmal lesions. Arch Ophthalmol 45:407437, 1916

41. Wolpert SM: Neuroradiology in Boston: historical beginnings. AJNR Am J Neuroradiol 16:1093-1098, 1995

\section{Disclosures}

The authors report no conflict of interest concerning the materials or methods used in this study or the findings specified in this paper.

\section{Author Contributions}

Conception and design: Pascual, Prieto. Acquisition of data: Pascual, Prieto. Analysis and interpretation of data: all authors. Drafting the article: all authors. Critically revising the article: Pascual. Reviewed submitted version of manuscript: Pascual, Prieto. Approved the final version of the manuscript on behalf of all authors: Pascual. Statistical analysis: Barrios.

\section{Supplemental Information Companion Papers}

Prieto R, Pascual JM, Barrios L: Harvey Cushing's craniopharyngioma treatment: Part 2. Surgical strategies and results of his pioneering series. DOI: 10.3171/2018.5.JNS18154.

\section{Correspondence}

José María Pascual: La Princesa University Hospital, Madrid, Spain.jmpasncj@hotmail.com. 